\title{
The Feynman Representation for the Dirac Propagator with a Radially Symmetric Potential
}

By

\author{
Brian JEFFERIES*
}

\begin{abstract}
The dynamical group associated with the Dirac equation with a radially symmetric potential in four space-time dimensions is represented in terms of integrals with respect to operator valued set functions associated with the free Dirac operator. In coordinates in which $c=\hbar=1$, the class of potentials treated includes Coulomb potentials $-a / r$ with $|a|<1$.
\end{abstract}

\section{Introduction}

This paper is a continuation of work commenced by the author in [J2], on the representation of the dynamical group $U(t)$ associated with the Dirac equation with a radially symmetric potential as an integral with respect to an operator valued set function $M_{t}$, for each $t>0$. The operator valued set functions $\left\langle M_{t}\right\rangle_{t \geq 0}$, constructed from the free Dirac group $S$ and a spectral measure $Q_{r}$ of multiplication by characteristic functions, measure the random events of an underlying one dimensional process in place of a probability measure.

For a radially symmetric potential $V: x \mapsto q(|x|), x \in \mathbb{R}^{3}$, the representation has the form

$$
U(t)=\int_{\Omega} \exp \left[-i \int_{0}^{t} q(\omega(s)) d s\right] d M_{t}(\omega), \quad t>0 .
$$

The notation of the present work differs from that of [J2] in that the sample space $\Omega$ is taken to be a set of paths $\omega:[0, \infty) \rightarrow[0, \infty)$ rather than a collection of paths with values in $\mathbb{R}^{3}$. It is proved in $[\mathrm{J} 2$, Theorem 4.4$]$ that

Communicated by H. Araki, May 15, 1995.

* 1991 Mathematics Subject Classifications: Primary 81S40, 47D06; Secondary 28B05, 28C20, $35 \mathrm{~L} 45$.

School of Mathematics, UNSW, NSW 2052, Australia. 
the space $\Omega$ may be taken to be the collection of all paths $\omega:[0, \infty) \rightarrow[0, \infty)$ with speed \pm 1 and at most finitely many changes of direction in any bounded time interval. The feature of the "Zitterbewegung" of the Dirac particle remarked on elsewhere [I-T] is also present in four space-time dimensions.

The representation $(0.1)$ is proved in [ $\mathrm{J} 2$, Theorem 6.3] under the assumption that the function $q$ is locally integrable on $[0, \infty)$ and locally square integrable on $(0, \infty)$, a result which excludes the case of a Coulomb potential $q(r)=-\beta / r$. The difficulty encountered in proving $(0.1)$ for a Coulomb potential $q$ is that for paths $\omega$ which hit the origin during the time interval $[0, t]$, the integral $\int_{0}^{t} q(\omega(s)) d s$ diverges logarithmically. The main result of the present work, Corollary 4.8, establishes that the set of such paths is negligible for $M_{t}$, contrary to the (unproved) assertion in the final paragraph of [J2, p 298], so at least the integrand in (0.1) makes sense for a full set of paths $\omega \in \Omega$. The notion of negligible sets for the unbounded set function $M_{t}$ and an interpretation of the integral (0.1) are outlined in [J2, Section 5].

The representation $(0.1)$ is proved for functions $q:(0, \infty) \rightarrow \mathbb{R}$ which satisfy the bound

$$
q=q^{(1)}+q^{(2)}, \sup _{r>0}\left|q^{(1)}(r)\right| r \leq \mu, \sup _{r>0}\left|q^{(2)}(r)\right|<\infty
$$

where $\mu<\sqrt{3} / 2$. In particular, the result applies to Coulomb potentials $q(r)=-a / r, r>0$ with $|a|<\sqrt{3} / 2$. Here we are working in a coordinate system in which the speed of light $c$ and Planck's constant $\hbar$ are equal to one, so that $\mu<1$ corresponds to $Z<137$ in atomic units.

Section 1 establishes some terminology for the type of processes this work employs. In Section 2, the radially symmetric Dirac process is formally defined and some results concerning the process proved in [ $\mathrm{J} 2]$ are mentioned again. Section 3 reviews the notion, also developed in [J2], of integration with respect to the unbounded set functions associated with the radially symmetric Dirac process. Depending on the reader's willingness to believe the author's assertions that are not herein proved, the present paper can be read independently of [J2]. The main arguments are in Section 4. The result that the set of paths which hit the origin is $M_{t}^{+}$-null is proved in Corollary 4.8 after obtaining, in Proposition 4.4, an explicit representation by a perturbation series expansion, for regularisations $M_{t}^{(\varepsilon)}, \varepsilon>0$, of $M_{t}$. The main result is given in Section 5. The representation (0.1) is established in Theorem 5.4 for potentials satisfying condition (0.2) with $\mu<\sqrt{3} / 2$. For potentials $q$ satisfying (0.2) with $\mu<1$, and having constant sign near the origin, the dynamical group $U(t)$ is represented in Theorem 5.6 as the limit of integrals like that on the right hand side of equation (0.1). 


\section{§1. $(S, Q)$-Processes}

In this section, we outline an abstract framework to deal with the processes we wish to consider in the remainder of the paper. The framework indicates the relationship between what we consider a "process" here, and the more familiar notion of a stochastic process with respect to a probability measure space. Let $(\Sigma, \mathscr{B})$ be a measurable space and let $S$ be a semigroup of continuous linear operators acting on a Banach space $Y$. The space $\mathscr{L}(Y)$ of continuous linear operators acting on $Y$ is equipped with the strong operator topology. Then $S$ is a map from $\mathbb{R}_{+}$into the space $\mathscr{L}(Y)$ such that $S(s+t)=S(s) S(t)$ for all $s, t \geq 0$, and $S(0)=I d$, the identity operator. No continuity assumption is made on $S$ at this stage. Suppose that $Q: \mathscr{B} \rightarrow \mathscr{L}(Y)$ is a spectral measure, that is, $Q$ is an operator valued measure, $\sigma$-additive for the strong operator topology, such that $Q(\Sigma)=I d$ and $Q(A \cap B)=Q(A) Q(B)$ for all $A, B \in \mathscr{B}$.

Suppose that $\Omega$ is some non-empty collection of functions $\omega: \mathbb{R}_{+} \rightarrow \Sigma$. Let $t>0, n=1,2, \ldots, 0<t_{1}<\cdots<t_{n}<t$ and suppose that $B_{1}, \ldots, B_{n} \in \mathscr{B}$ are subsets of $\Sigma$. For each subset $E$ of $\Omega$ of the form

$$
E=\left\{\omega \in \Omega: \omega\left(t_{1}\right) \in B_{1}, \ldots, \omega\left(t_{n}\right) \in B_{n}\right\},
$$

the operator $M_{t}(E) \in \mathscr{L}(Y)$ is defined by the formula

$$
M_{t}(E)=S\left(t-t_{n}\right) Q\left(B_{n}\right) S\left(t_{n}-t_{n-1}\right) \cdots Q\left(B_{2}\right) S\left(t_{2}-t_{1}\right) Q\left(B_{1}\right) S\left(t_{1}\right) .
$$

Provided that the set $E$ is non-empty whenever each of the sets $B_{1}, \ldots, B_{n}$ is not a $Q$-null set, it follows that as the times $t_{1}, \ldots, t_{n}$, the sets $B_{1}, \ldots, B_{n}$ and $n=1,2, \ldots$ vary, but $t$ is fixed, the sets $E$ form a semi-algebra $\mathscr{S}_{t}$ of subsets of $\Omega$ and the expression (1.2) defines an additive operator valued set function $M_{t}$, defined on $\mathscr{S}_{t}$ and acting on $Y$. Furthermore, the additivity of the set function $M_{t}$ ensures that it has a unique extension, also denoted by $M_{t}$, to the algebra $\alpha\left(\mathscr{S}_{t}\right)$ of subsets of $\Omega$ generated by $\mathscr{T}_{t}$. Set $X_{s}(\omega)=\omega(s)$ for all $s \geq 0$. Elements of the semi-algebra $\mathscr{S}_{t}$ are called elementary events before time $t$. Then the collection $\left(\Omega,\left\langle\mathscr{S}_{t}\right\rangle_{t \geq 0},\left\langle M_{t}\right\rangle_{t \geq 0} ;\left\langle X_{t}\right\rangle_{t \geq 0}\right)$ is called an $(S, Q)$-process. The same terminology is adopted in the case that the semi-algebras $\left\langle\mathscr{S}_{t}\right\rangle_{t \geq 0}$ are replaced by the $\sigma$-algebras that they generate and for each $t>0, M_{t}$ is an operator valued measure. The Banach space $Y$ is called the state space of the process. For a Markov process with probabilities $P^{x}, x \in \mathbb{R}^{d}$, the associated operator valued measures $W_{t}: \mathscr{S}_{t} \rightarrow \mathscr{L}\left(L^{p}\left(\mathbb{R}^{d}\right)\right)$ are defined for $A \in \mathscr{S}_{t}$ by the formula

$$
\left\langle W_{t}(A) \phi, \psi\right\rangle=\int_{\mathbb{R}^{d}} P^{x}\left(\chi_{A}\left(\psi \circ X_{t}\right)\right) \phi(x) d x, \quad \phi \in L^{p}\left(\mathbb{R}^{d}\right), \psi \in L^{p^{\prime}}\left(\mathbb{R}^{d}\right) .
$$


The idea of associating an operator valued set function with an arbitrary semigroup and a spectral measure is due to I. Kluvánek [Klu].

If the range $\left\{M_{t}(A): A \in \alpha\left(\mathscr{S}_{t}\right)\right\}$ of the additive set function $M_{t}$ on the algebra $\alpha\left(\mathscr{S}_{t}\right)$ is bounded in the uniform operator norm of $\mathscr{L}(Y)$ and $\Omega=\Sigma^{\mathbb{R}+}$, then under certain mild conditions, $M_{t}$ is actually the restriction to $\alpha\left(\mathscr{S}_{t}\right)$ of an operator valued measure defined on the $\sigma$-algebra $\sigma\left(\mathscr{S}_{t}\right)$ generated by $\mathscr{S}_{t}$-the techniques of probability theory are directly applicable to this case. If the range of $M_{t}$ is unbounded on $\alpha\left(\mathscr{S}_{t}\right)$ in the uniform operator norm of $\mathscr{L}(Y)$, then integration with respect to $M_{t}$ may be controlled by a family of operator valued measures; this is the situation for the radially symmetric Dirac process considered in the next section, and discussed in greater detail in Section 3.

\section{§2. The Radially Symmetric Dirac Process}

The free Dirac operator is defined in $L^{2}\left(\mathbb{R}^{3} ; \mathbb{C}^{4}\right)$ by means of the differential expression $D=c \sum_{j=1}^{3} \alpha_{j} p_{j}+\alpha_{4} m_{a} c^{2}$, where $c>0$ is the velocity of light, $m_{a}>0$ is the mass of the particle, $p_{j}=\frac{\hbar}{i} \frac{\partial}{\partial x_{j}}$, and

$$
\alpha_{j}=\left(\begin{array}{cc}
0 & \sigma_{j} \\
\sigma_{j} & 0
\end{array}\right), j=1,2,3, \quad \alpha_{4}=\left(\begin{array}{cc}
\sigma^{0} & 0 \\
0 & -\sigma_{0}
\end{array}\right) .
$$

Here $\sigma_{1}, \sigma_{2}, \sigma_{2}$ are the Pauli matrices

$$
\sigma_{1}=\left(\begin{array}{ll}
0 & 1 \\
1 & 0
\end{array}\right), \quad \sigma_{2}=\left(\begin{array}{cc}
0 & -i \\
i & 0
\end{array}\right), \quad \sigma_{3}=\left(\begin{array}{cc}
1 & 0 \\
0 & -1
\end{array}\right)
$$

and $\sigma_{0}=\left(\begin{array}{ll}1 & 0 \\ 0 & 1\end{array}\right)$ is the $2 \times 2$ identity matrix.

Then $D$ defines a selfadjoint operator, and so, a unitary group $S_{D}(t)=e^{i D t}$, $t \in \mathbb{R}$ of operators acting on $L^{2}\left(\mathbb{R}^{3} ; \mathbb{C}^{4}\right)$. For any $n=1,2, \ldots$, the space $\mathbb{C}^{n}$ is assumed to be equipped with the inner product $\langle a, b\rangle=\sum_{j=1}^{n} a_{j} \bar{b}_{j}$ for $a=\left(a_{1}, \ldots, a_{n}\right)$ and $b=\left(b_{1}, \ldots, b_{n}\right)$ in $\mathbb{C}^{n}$. The space $L^{2}\left(\mathbb{R}^{3} ; \mathbb{C}^{4}\right)$ is a Hilbert space with the innerproduct $(f, g)=\int_{\mathbb{R}^{3}}\langle f(x), g(x)\rangle d x$ for $f, g \in L^{2}\left(\mathbb{R}^{3} ; \mathbb{C}^{4}\right)$. We choose a coordinate system in which $c=\hbar=1$.

The space of all smooth $\mathbb{C}^{2}$-valued functions on $\mathbb{R}_{++}=(0, \infty)$ with compact support is denoted by $C_{c}^{\infty}\left(\mathbb{R}_{++}, \mathbb{C}^{2}\right)$. The Borel $\sigma$-algebra of a Hausdorff topological space $\Sigma$ is denoted by $\mathscr{B}(\Sigma)$.

By virtue of the angular momentum decomposition of the Dirac operator, there exists a family $\mathscr{H}_{k, m}, k= \pm 1, \pm 2, \ldots$ and $m=-|k|,-|k|+1, \ldots,|k|-1$ of mutually orthogonal subspaces of $L^{2}\left(\mathbb{R}^{3}, \mathbb{C}^{4}\right)$, such that $L^{2}\left(\mathbb{R}^{3} ; \mathbb{C}^{4}\right)$ is the Hilbert space direct sum of $\mathscr{H}_{k, m}$, each space $\mathscr{H}_{k, m}$ is a reducing subspace for the free Dirac operator $D$ and in each $\mathscr{H}_{k, m}, D$ is unitarily equivalent to the 
closure in $L^{2}\left(\mathbb{R}_{+} ; \mathbb{C}^{2}\right)$, of the operator $\tau_{k}$ defined by

$$
\tau_{k}: g \mapsto\left(\begin{array}{cc}
0 & -1 \\
1 & 0
\end{array}\right)\left(\begin{array}{l}
g_{1}^{\prime}(r) \\
g_{2}^{\prime}(r)
\end{array}\right)+\left(\begin{array}{cc}
m_{a} & -k r^{-1} \\
-k r^{-1} & -m_{a}
\end{array}\right)\left(\begin{array}{l}
g_{1}(r) \\
g_{2}(r)
\end{array}\right)
$$

on the space $C_{c}^{\infty}\left(\mathbb{R}_{++} ; \mathbb{C}^{2}\right)$. The essential selfadjointness of the operator $D$ with domain $C_{c}^{\infty}\left(\mathbb{R}^{3} \backslash\{0\} ; \mathbb{C}^{4}\right)$ serves to establish the essential selfadjointness in $L^{2}\left(\mathbb{R}_{+} ; \mathbb{C}^{2}\right)$ of the operator $\tau_{k}$ for each $k= \pm 1, \pm 2, \ldots$. The details are laid out in [A]. Thus, for each $k= \pm 1, \pm 2, \ldots$ and $m=-|k|,-|k|+1, \ldots$, $|k|-1$, we have $\tau_{k}=\mathscr{U}_{k, m}\left(D\left\lceil\mathscr{H}_{k, m}\right) \mathscr{U}_{k, m}^{*}\right.$ for a unitary operator $\mathscr{U}_{k, m}: \mathscr{H}_{k, m} \rightarrow$ $L^{2}\left(\mathbb{R}_{+} ; \mathbb{C}^{2}\right)$ onto a closed subspace of $L^{2}\left(\mathbb{R}_{+} ; \mathbb{C}^{2}\right)$. Here $D\left\lceil\mathscr{H}_{k, m}\right.$ is the restriction of $D$ to the reducing subspace $\mathscr{H}_{k, m}$.

For each $k \in \mathbb{Z} \backslash\{0\}$, let $T_{k}$ be the unitary group of operators defined on $L^{2}\left(\mathbb{R}_{+} ; \mathbb{C}^{2}\right)$ by $T_{k}(t)=e^{i \tau_{k} t}$ for all $t \in \mathbb{R}$. Let $Q$ be the spectral measure, acting on $L^{2}\left(\mathbb{R}_{+} ; \mathbb{C}^{2}\right)$, of multiplication by the characteristic functions of Borel subsets of $\mathbb{R}_{+}$.

Let $\Omega$ be the set of all continuous functions $\omega: \mathbb{R}_{+} \rightarrow \mathbb{R}_{+}$such that for each $t>0$, the sets $\left\{\omega^{\prime}=1\right\}$ and $\left\{\omega^{\prime}=-1\right\}$ in $(0, t)$ are the finite union of open intervals, the union of which contains all except finitely many points in $(0, t)$. Less formally, the paths $\omega \in \Omega$ are continuous with velocity \pm 1 , and only finitely many changes in direction in any bounded time interval.

For each $k= \pm 1, \pm 2, \ldots$, let $\left(\Omega,\left\langle\mathscr{S}_{t}\right\rangle_{t \geq 0},\left\langle R_{t}^{(k)}\right\rangle_{t \geq 0} ;\left\langle X_{t}\right\rangle_{t \geq 0}\right)$ be the $\left(T_{k}, Q\right)$-process with state space $L^{2}\left(\mathbb{R}_{+} ; \mathbb{C}^{2}\right)$. For each $t \geq 0, \mathscr{S}_{t}$ is the algebra generated by the family of all elementary events before time $t, R_{t}^{(k)}: \mathscr{S}_{t} \rightarrow$ $\mathscr{L}_{s}\left(L^{2}\left(\mathbb{R}_{+} ; \mathbb{C}^{2}\right)\right)$ is an additive operator valued set function and $X_{t}(\omega)=\omega(t)$, $\omega \in \Omega$ is the evaluation map.

The $\left(S_{D}, Q_{r}\right)$-process with state space $L^{2}\left(\mathbb{R}^{3} ; \mathbb{C}^{4}\right)$ is denoted by

$$
\left(\Omega,\left\langle\mathscr{T}_{t}\right\rangle_{t \geq 0},\left\langle M_{t}\right\rangle_{t \geq 0} ;\left\langle X_{t}\right\rangle_{t \geq 0}\right) .
$$

For each $t \geq 0, M_{t}: \mathscr{S}_{t} \rightarrow \mathscr{L}_{s}\left(L^{2}\left(\mathbb{R}^{3} ; \mathbb{C}^{4}\right)\right)$ is an additive operator valued set function. The $\left(S_{D}, Q_{r}\right)$-process is called the radially symmetric Dirac process. The notation adopted here differs from that of [J2, p 317]; here we wish to emphasise the one-dimensional character of the process.

The radially symmetric Dirac process is written in terms of the processes

$$
\left(\Omega,\left\langle\mathscr{S}_{t}\right\rangle_{t \geq 0},\left\langle R_{t}^{(k)}\right\rangle_{t \geq 0} ;\left\langle X_{t}\right\rangle_{t \geq 0}\right), \quad k= \pm 1, \pm 2, \ldots
$$

by setting

$$
M_{t}(E)=\sum_{|k|=1}^{\infty} \oplus\left(\sum_{m=-|k|}^{|k|-1} \oplus \mathscr{U}_{k, m}^{*} R_{t}^{(k)}(E) \mathscr{U}_{k, m}\right) \quad \text { for all } E \in \mathscr{S}_{t}
$$




\section{§3. Integration with Respect to $M_{t}$ and $\left\langle M_{i}^{(\varepsilon)}\right\rangle_{\varepsilon}>0$}

In order to define integration with respect to the operator valued set functions $M_{t}$, we define $\sigma$-additive operator valued measures $M_{t}^{(\varepsilon)}$ for all $\varepsilon>0$ by cutting off the singularity in the right hand side of formula (2.1) at $r=0$. The details of the following construction are laid out in [J2].

Let $N=1 / \sqrt{2}\left(\begin{array}{ll}1 & i \\ i & 1\end{array}\right)$. Then $i N^{*}\left(\begin{array}{cc}0 & -1 \\ 1 & 0\end{array}\right) N=\left(\begin{array}{rr}1 & 0 \\ 0 & -1\end{array}\right)$. Let $\mathscr{D}(\tau)$ be the collection of all functions $f \in L^{2}\left(\mathbb{R}_{+} ; \mathbb{C}^{2}\right)$ such that $f$ is absolutely continuous on all bounded subintervals of $\mathbb{R}_{+}, f^{\prime} \in L^{2}\left(\mathbb{R}_{+} ; \mathbb{C}^{2}\right)$ and $\left(N^{*} f\right)_{2}(0)=0$. Let $\tau: \mathscr{D}(\tau) \rightarrow L^{2}\left(\mathbb{R}_{+} ; \mathbb{C}^{2}\right)$ be the operator $\left(\begin{array}{cc}0 & -1 \\ 1 & 0\end{array}\right) d / d x$. Then $i \tau$ is the generator of a $\mathrm{C}_{0}$-semigroup on $L^{2}\left(\mathbb{R}_{+} ; \mathbb{C}^{2}\right)$.

For every $\varepsilon \geq 0$, let

$$
U_{k, \varepsilon}(r)=\left\{\begin{array}{cc}
\left(\begin{array}{cc}
m_{a} & -k r^{-1} \\
-k r^{-1} & -m_{a}
\end{array}\right) & \text { for all } r>\varepsilon, \\
\left(\begin{array}{cc}
m_{a} & -k \varepsilon^{-1} \\
-k \varepsilon^{-1} & -m_{a}
\end{array}\right) & \text { for all } 0<r \leq \varepsilon .
\end{array}\right.
$$

For each $\varepsilon>0, U_{k, \varepsilon}$ is a bounded function with values in the $2 \times 2$ hermitian matrices and $i \tau_{k, \varepsilon}=i\left(\tau+U_{k, \varepsilon}\right)$ is the generator of a $\mathrm{C}_{0}$-semigroup $[\mathrm{K} 1$, Theorem IX.2.1].

The operator defined by (2.1) is essentially selfadjoint; its closure in $L^{2}\left(\mathbb{R}_{+} ; \mathbb{C}^{2}\right)$ is denoted by the same symbol. Then $\lim _{\varepsilon \rightarrow 0} e^{i \tau_{k, \varepsilon} t}=e^{i \tau_{k} t}$ in the strong operator topology of $\mathscr{L}\left(L^{2}\left(\mathbb{R}_{+} ; \mathbb{C}^{2}\right)\right)[\mathrm{J} 2, \mathrm{p} 309]$.

Let $\mathscr{R}_{t}$ be the $\sigma$-algebra generated by elementary events in $\Omega$ before time t. It turns out ([J2, Theorem 4.3]; see also Corollary 4.6 below) that for each $k= \pm 1, \pm 2, \ldots$ and each $\varepsilon>0$, there exists an $\left(e^{i \tau_{k, \varepsilon^{*}}}, Q\right)$-process

$$
\left(\Omega,\left\langle\mathscr{R}_{t}\right\rangle_{t \geq 0},\left\langle R_{t}^{(k, \varepsilon)}\right\rangle_{t \geq 0} ;\left\langle X_{t}\right\rangle_{t \geq 0}\right)
$$

for which $R_{t}^{(k, \varepsilon)}: \mathscr{R}_{t} \rightarrow L^{2}\left(\mathbb{R}_{+} ; \mathbb{C}^{2}\right)$ is a $\sigma$-additive operator valued measure for each $t \geq 0$. The measures $R_{t}^{(k, \varepsilon)}, \varepsilon>0$ will be used to control integration with respect to $R_{t}^{(k)}$.

Let $\varepsilon>0$. The finite direct $\operatorname{sum} \sum_{k, m \in K_{\varepsilon}} \oplus \mathscr{U}_{k, m}^{*}\left(\tau+U_{k, \varepsilon}\right) \mathscr{U}_{k, m}$ over the set $K_{\varepsilon}$ of all integers $k, m$ such that $m=-|k|,-|k|+1, \ldots,|k|-1$ and $1 \leq|k| \leq 1 / \varepsilon$ is denoted by $D_{\varepsilon}$. Then $i D_{\varepsilon}$ is the generator of a $\mathrm{C}_{0}$-contraction semigroup $S_{D_{\varepsilon}}$ on $L^{2}\left(\mathbb{R}^{4} ; \mathbb{C}^{4}\right)$ satisfying

$$
S_{D_{\varepsilon}}(t)=\sum_{k, m \in K_{\varepsilon}} \oplus \mathscr{U}_{k, m}^{*} e^{i \tau_{k, \varepsilon} t} \mathscr{U}_{k, m}, \quad \text { for all } t \geq 0 .
$$

The range $K_{\varepsilon}$ of the values of the integers $k, m$ in the direct sum is not mentioned explicitly in [J2]; taking a finite sum ensures that there is no difficulty with the exponentially increasing bound [J2, Lemma 3.1] on the 
diameters of the ranges of $R_{t}^{(k, \varepsilon)}$ for increasing $|k|$. Because of the boundary conditions imposed on $\tau$, the operator $D_{\varepsilon}$ is not selfadjoint. Nevertheless, as $\varepsilon \rightarrow 0^{+}$, the closed unbounded operator $D_{\varepsilon}$ converges in the strong resolvent sense to the free Dirac operator $D$.

For each $\varepsilon>0$, there exists an $\left(S_{D_{\varepsilon}}, Q_{r}\right)$-process

$$
\left(\Omega,\left\langle\mathscr{R}_{t}\right\rangle_{t \geq 0},\left\langle M_{t}^{(\varepsilon)}\right\rangle_{t \geq 0} ;\left\langle X_{t}\right\rangle_{t \geq 0}\right)
$$

such that

$$
M_{t}^{(\varepsilon)}(E)=\sum_{k, m \in K_{\varepsilon}} \oplus \mathscr{U}_{k, m}^{*} R_{t}^{(k, \varepsilon)}(E) \mathscr{U}_{k, m} \quad \text { for all } E \in \mathscr{R}_{t} .
$$

Let $t>0$. Rather than formally define the idea of a function being integrable with respect to $M_{t}$ relative to the family $\left\langle M_{t}^{(\varepsilon)}\right\rangle_{\varepsilon>0}$ of operator valued measures, we shall sketch the main idea and refer to [J2, Section 5], where the details are laid out explicitly.

The space $L^{1}\left(M_{t}^{+}\right)$denotes the vector space of all (equivalence classes of) functions which are integrable with respect to each operator valued measure $M_{t}^{(\varepsilon)}, \varepsilon>0$. A net converges in $L^{1}\left(M_{t}^{+}\right)$exactly when it converges in each space $L^{1}\left(M_{t}^{(\varepsilon)}\right), \varepsilon>0$.

For a simple function $s$ based on the algebra $\mathscr{S}_{t}$, the operator valued set function $s \cdot M_{t}: \mathscr{S}_{t} \rightarrow \mathscr{L}\left(L^{2}\left(\mathbb{R}^{3} ; \mathbb{C}^{4}\right)\right)$ - the indefinite integral of $s$ with respect to $M_{t}$ - is defined by linearity, in the obvious way; this is possible, because $M_{t}: \mathscr{S}_{t} \rightarrow \mathscr{L}\left(L^{2}\left(\mathbb{R}^{3} ; \mathbb{C}^{4}\right)\right)$ is additive. Suppose that $\mathfrak{I}_{t}$ is the locally convex topology defined on the simple functions such that a net $\left\langle s_{l}\right\rangle_{l \in I}$ converges to a simple function $s$ if and only if $\left\langle s_{l}\right\rangle_{l \in I}$ converges to $s$ in $L^{1}\left(M_{t}^{+}\right)$, and for each $A \in \mathscr{S}_{t}$, the net $\left\langle\left(s_{l} \cdot M_{t}^{(\varepsilon)}\right)(A)\right\rangle_{l \in I}$ converges to the integral $\left(s \cdot M_{t}^{(\varepsilon)}\right)(A)$ of $s$ with respect to $M_{t}^{(\varepsilon)}$ over $A$, in the weak operator topology, uniformly for $0<\varepsilon \leq 1$. Then given a bounded Cauchy net $\left\langle s_{l}\right\rangle_{l \in I}$ for the topology $\mathfrak{I}_{t}$, there exists $f \in L^{1}\left(M_{t}^{+}\right)$such that $\left\langle s_{l}\right\rangle_{l \in I}$ converges to $f$ in $L^{1}\left(M_{t}^{+}\right)$, and for each $A \in \mathscr{S}_{t}$, the net $\left\langle\left(s_{l} \cdot M_{t}^{(\varepsilon)}\right)(A)\right\rangle_{l \in I}$ converges to $\left(f \cdot M_{t}^{(\varepsilon)}\right)(A)$ in the weak operator topology, uniformly for $0<\varepsilon \leq 1$. Moreover,

$$
\int_{A} f(\omega) d M_{t}(\omega)=\left(f \cdot M_{t}\right)(A):=\lim _{\varepsilon \rightarrow 0+}\left(f \cdot M_{t}^{(\varepsilon)}\right)(A)
$$

exists in the weak operator topology for each $A \in \mathscr{S}_{t}$. Then $f \cdot M_{t}: \mathscr{S}_{t} \rightarrow$ $\mathscr{L}\left(L^{2}\left(\mathbb{R}^{3} ; \mathbb{C}^{4}\right)\right)$ is an additive operator valued set function defined on the algebra $\mathscr{S}_{t}$ and the function $f$ is said to be $M_{t}^{+}-M_{t}$-integrable. The space of all (equivalence classes of) $M_{t}^{+}-M_{t}$-integrable functions is denoted by $L^{1}\left(M_{t}^{+}, M_{t}\right)$. It is endowed with the topology associated with $\mathfrak{I}_{t}$. With this viewpoint, the null sets of $M_{t}$ are just those subsets of $\Omega$ which are $M_{t}^{(\varepsilon)}$-null sets for each $\varepsilon>0$. 
Remark. The idea of integration with respect to unbounded set functions $M_{t}$ outlined above applies to situations more general than the radially symmetric Dirac process. The basic requirement is obviously that $M_{t}$ should belong to the closure in the topology of setwise convergence on the underlying semi-algebra of elementary events, of a set $\mathscr{M}_{t}=\left\{M_{t}^{(\varepsilon)}: \varepsilon>0\right\}$ of $\sigma$-additive measures. In that situation, the scheme outlined in [J1] applies, and we obtain the notion of $\mathscr{M}_{t}-M_{t}$-integrable functions. There may be many "regularisations" $\mathscr{M}_{t}$ of $M_{t}$ - the appropriate one is given by the problem at hand. For the radial parts of the Dirac operator, we eliminated the singularity at zero to obtain the family $\mathscr{M}_{t}$ above.

By contrast, in non-relativistic quantum mechanics [J1] and, presumably, quantum field theory in Minkowski space, the unbounded set functions $M_{t}^{m}, m \in \mathbb{R}, m \neq 0$ are the boundary values on the real axis of set functions $M_{t}^{z}$ with $z$ in the upper half-plane $P_{+}$. The family $\mathscr{M}_{t}=\left\{M_{t}^{a i}: a>0\right\}$ of $\sigma$-additive measures serves to define $\mathscr{M}_{t}-M_{t}$-integrable functions. Hence, the "regularisation" $\mathscr{M}_{t}$ of the set function $M_{t}$ is obtained by analytic continuation $M_{t}^{z}, z \in P_{+}$to the upper half plane, followed by restriction to the positive imaginary axis.

The technique of regularisation is familiar from other areas of analysis and is naturally applied to the treatment of integration with respect to the unbounded set function arising in quantum physics.

\section{§4. The Support of the Cut-off Measures $\mathbb{R}_{t}^{(k, \varepsilon)}, \varepsilon>0$}

We shall require more information about the support of the operator valued measures $R_{t}^{(k, \varepsilon)}, \varepsilon>0, k= \pm 1, \pm 2, \ldots$ To this end, the $\mathrm{C}_{0}$-semigroup $e^{i\left(\tau+U_{k, \varepsilon) t}\right.}$ can be written as a perturbation series $e^{i\left(\tau+U_{k, \varepsilon) t}\right.}=\sum_{j=0}^{\infty} i^{j} V_{j}^{(\varepsilon)}(t)$, with $V_{0}(t)=e^{i \tau t}$ and

$$
\begin{gathered}
V_{j}^{(\varepsilon)}(t)=\int_{0}^{t} \int_{0}^{\alpha_{J}} \cdots \int_{0}^{\alpha_{2}} e^{i \tau\left(t-\alpha_{\jmath}\right)} U_{k, \varepsilon} e^{i \tau\left(\alpha_{J}-\alpha_{\jmath}-1\right)} \ldots \\
e^{i \tau\left(\alpha_{2}-\alpha_{1}\right)} U_{k, \varepsilon} e^{i \tau \alpha_{1}} d \alpha_{1} \cdots d \alpha_{j}
\end{gathered}
$$

for all $t \geq 0$ and all $j=1,2, \ldots$. Here $U_{k, \varepsilon}$ is the operator of multiplication by the matrix valued function $r \mapsto U_{k, \varepsilon}(r), r \geq 0$. On applying this expansion to the definition of $R_{t}^{(k, \varepsilon)}$, we obtain $R_{t}^{(k, \varepsilon)}(A)=\sum_{J=0}^{\infty} i^{J} R_{t, J}^{(k, \varepsilon)}(A)$, for all $A \in \mathscr{R}_{t}$, where

$$
\begin{gathered}
R_{t, J}^{(k, \varepsilon)}(E)=\sum_{j_{0}+\cdots+j_{n}=J} V_{j_{n}}^{(\varepsilon)}\left(t-t_{n}\right) Q\left(B_{n}\right) V_{j_{n-1}}^{(\varepsilon)}\left(t_{n}-t_{n-1}\right) \cdots \\
V_{j_{1}}^{(\varepsilon)}\left(t_{2}-t_{1}\right) Q\left(B_{1}\right) V_{j_{0}}^{(\varepsilon)}\left(t_{1}\right),
\end{gathered}
$$

for all elementary events $E$ of the form (1.1), and all $J=0,1,2, \ldots$ The sum 
is over integers $j_{0}, \ldots, j_{n} \geq 0$.

It turns out that for each $J=0,1,2, \ldots, R_{t, J}^{(k, \varepsilon)}$ is a $\sigma$-additive operator valued measure on $\mathscr{R}_{t}\left[\mathrm{~J} 2\right.$, Lemma 4.2]. The set $\Omega_{t}$ denotes the collection of restrictions of paths $\omega \in \Omega$ to the interval $[0, t]$. It is natural to consider a set $A \in \mathscr{R}_{t}$ as being a subset of $\Omega_{t}$, via the restriction map. We shall pass between the two viewpoints without further mention.

The aim in this section is to show that the set $\Gamma_{t}$ of all paths $\omega \in \Omega$ which hit the origin at some time in the interval $[0, t]$ is an $R_{t}^{(k, \varepsilon)}$-null set for each $\varepsilon>0$. To this end, we shall show that $\Gamma_{t}$ is $R_{t, J}^{(k, \varepsilon)}$-null for each $\varepsilon>0$ and each $J=0,1,2, \ldots$.

Let $T$ be the infinitesimal generator of the semigroup $e^{T s}, s \geq 0$ which sends $\phi \in L^{2}\left(\mathbb{R}_{+} ; \mathbb{C}^{2}\right)$ to the function

$$
\left(e^{T s} \phi\right)(x)=\left(\begin{array}{c}
\phi_{1}(x+s) \\
\chi_{\mathbb{R}_{+}}(x-s) \phi_{2}(x-s)
\end{array}\right), \quad \text { for almost all } x \in \mathbb{R}_{+},
$$

that is, the domain $\mathscr{D}(T)$ of $T$ is the set of all functions $f \in L^{2}\left(\mathbb{R}_{+} ; \mathbb{C}^{2}\right)$ such that $f$ is absolutely continuous on all bounded subintervals of $\mathbb{R}_{+}$, $f^{\prime} \in L^{2}\left(\mathbb{R}_{+} ; \mathbb{C}^{2}\right)$ and $f_{2}(0)=0$. Then for the matrix $N$ defined in Section 3, the equality

$$
i \tau_{k, \varepsilon}=N\left[\left(\begin{array}{cc}
1 & 0 \\
0 & -1
\end{array}\right) \frac{d}{d x}+i N^{*} U_{k, \varepsilon} N\right] N^{*}=N\left[T+i N^{*} U_{k, \varepsilon} N\right] N^{*}
$$

holds, $\mathscr{D}(\tau)=N \mathscr{D}(T) N^{*}, i \tau=N T N^{*}$ and

$$
e^{i \tau_{k, \varepsilon} s}=N e^{\left(T+i N^{*} U_{k, \varepsilon} N\right) s} N^{*}, \quad \text { for all } s \geq 0 .
$$

The operator in $\mathscr{L}\left(L^{2}\left(\mathbb{R}_{+} ; \mathbb{C}^{2}\right)\right)$ of multiplication by $i N^{*} U_{k, \varepsilon} N$ is bounded, so $T+i N^{*} U_{k, \varepsilon} N$ is a bounded perturbation of the generator $T$ of a $\mathrm{C}_{0}$-semigroup, hence it is the generator of a $\mathrm{C}_{0}$-semigroup which has a "Dyson" series expansion [K1, Theorem IX.2.1].

The argument we are going to use is essentially combinatorial, so instead of working with the semigroups $e^{i \tau s}, s \geq 0$ and $e^{i \tau_{k, \varepsilon} s}, s \geq 0$, in formulae (4.1) and (4.2), we shall use $e^{T s}, s \geq 0$ and $e^{(T+U) s}, s \geq 0$, where $U$ is a nonzero constant matrix. The modifications required to replace $U$ by a matrix valued multiplication operator will become apparent from the following discussion. The situation in which formulae (4.1) and (4.2) apply can then be covered by the application of the similarity transformation $a \mapsto N a N^{*}$ acting on $\mathscr{L}\left(L^{2}\left(\mathbb{R}_{+} ; \mathbb{C}^{2}\right)\right)$.

To this end, set $e^{(T+U) t}=\sum_{j=0}^{\infty} V_{j}(t), V_{0}(t)=e^{T t}$ and

$$
V_{j}(t)=\int_{0}^{t} \int_{0}^{\alpha_{J}} \cdots \int_{0}^{\alpha_{2}} e^{T\left(t-\alpha_{J}\right)} U e^{T\left(\alpha_{J}-\alpha_{J}-1\right)} \cdots e^{T\left(\alpha_{2}-\alpha_{1}\right)} U e^{T \alpha_{1}} d \alpha_{1} \cdots d \alpha_{j}
$$


for all $t \geq 0$ and all $j=1,2, \ldots$. Here the same symbol $U$ denotes the operator acting on $L^{2}\left(\mathbb{R}_{+} ; \mathbb{C}^{2}\right)$ of pointwise multiplication by the constant matrix $U=\left(\begin{array}{ll}u_{11} & u_{12} \\ u_{21} & u_{22}\end{array}\right)$. We define $R_{t}(A)=\sum_{J=0}^{\infty} R_{t, J}(A)$ for all $A \in \mathscr{R}_{t}$, where

$$
\begin{aligned}
& R_{t, J}(E)=\sum_{j_{0}+\cdots+j_{n}=J} V_{j_{n}}\left(t-t_{n}\right) Q\left(B_{n}\right) V_{j_{n-1}}\left(t_{n}-t_{n-1}\right) \cdots \\
& V_{j_{2}}\left(t_{3}-t_{2}\right) Q\left(B_{2}\right) V_{j_{1}}\left(t_{2}-t_{1}\right) Q\left(B_{1}\right) V_{j_{0}}\left(t_{1}\right),
\end{aligned}
$$

for all elementary events $E$ of the form (1.1). That $R_{t, J}$ is well-defined and additive follows from the formula $\sum_{l+j=m} V_{l}(u) V_{j}(v)=V_{m}(u+v)$ for $m=0,1, \ldots$ and $u, v \geq 0[\mathrm{~J} 2,(4.2)]$. The sum is over all non-negative integers.

We shall compute $R_{t, 0}$ and $R_{t, 1}$ explicitly. The general nature of $R_{t, J}$ and hence, of $R_{t, J}^{(k, \varepsilon)}$, will then be apparent for all $J=0,1,2, \ldots$; a formal proof of Corollary 4.8 by induction would be less than illuminating.

First, for every set $E$ of the form (1.1),

$$
R_{t, 0}(E)=V_{0}\left(t-t_{n}\right) Q\left(B_{n}\right) V_{0}\left(t_{n}-t_{n-1}\right) \cdots V_{0}\left(t_{2}-t_{1}\right) Q\left(B_{1}\right) V_{0}\left(t_{1}\right),
$$

so that $R_{t, 0}$ is the $\left(e^{T}, Q, t\right)$-set function.

Let $\theta$ be the characteristic function of $\mathbb{R}_{+}$. It proves convenient in the following formulae to write the composite function $f \circ g$ as $f(g(\mathbb{x}))$ and $f$ as $f(\mathrm{x})$ on $\mathbb{R}_{+}$. Then for every $\phi \in L^{2}\left(\mathbb{R}_{+} ; \mathbb{C}^{2}\right)$, we have

$$
\begin{aligned}
R_{t, 0}(E) \phi & =e^{T\left(t-t_{n}\right)} Q\left(B_{n}\right) e^{T\left(t_{n}-t_{n}-1\right)} \cdots e^{T\left(t_{2}-t_{1}\right)}\left(\begin{array}{c}
\chi_{B_{1}}(\mathbb{x}) \phi_{1}\left(\mathbb{x}+t_{1}\right) \\
\chi_{B_{1}}(\mathrm{x}) \theta\left(\mathrm{x}-t_{1}\right) \phi_{2}\left(\mathrm{x}-t_{1}\right)
\end{array}\right) \\
& \vdots \\
& =\left(\begin{array}{c}
\chi_{B_{n}}\left(\mathrm{x}+t-t_{n}\right) \cdots \chi_{B_{1}}\left(\mathrm{x}+t-t_{1}\right) \phi_{1}(\mathrm{x}+t) \\
\chi_{B_{n}}\left(\mathrm{x}-t+t_{n}\right) \cdots \chi_{B_{1}}\left(\mathrm{x}-t+t_{1}\right) \theta(\mathrm{x}-t) \phi_{2}(\mathrm{x}-t)
\end{array}\right) .
\end{aligned}
$$

For each $x \geq t$ let $\gamma_{x, t}:[0, t] \rightarrow \mathbb{R}_{+}$be the path defined by $\gamma_{x, t}(s)=x-t+s$ for all $0 \leq s \leq t$. For each $x \geq 0$ let $\xi_{x, t}:[0, t] \rightarrow \mathbb{R}_{+}$be the path defined by $\xi_{x, t}(s)=x+t-s$ for all $0 \leq s \leq t$. Then in terms of the paths $\gamma_{x, t}$ and $\xi_{x, t}$, we have

$$
\left(R_{t, 0}(E) \phi\right)(x)=\left(\begin{array}{c}
\left.\chi_{B_{n}}\left(\xi_{x, t}\left(t_{n}\right)\right) \cdots \chi_{B_{1}}\left(\xi_{x, t}\left(t_{1}\right)\right) \phi_{1}\left(\xi_{x, t}(0)\right)\right) \\
\chi_{B_{n}}\left(\gamma_{x, t}\left(t_{n}\right)\right) \cdots \chi_{B_{1}}\left(\gamma_{x, t}\left(t_{1}\right)\right) \theta\left(\gamma_{x, t}(0)\right) \phi_{2}\left(\gamma_{x, t}(0)\right)
\end{array}\right),
$$

for almost all $x \geq 0$. Let $\delta_{\gamma}: \mathscr{R}_{t} \rightarrow\{0,1\}$ be the unit point mass as the path $\gamma \in \Omega_{t}$; as mentioned earlier, we consider elements of $\mathscr{R}_{t}$ to be subsets of $\Omega_{t}$. On appealing to the $\sigma$-additivity of $R_{t, 0}$, we have proved the following

Proposition 4.1 . For all $A \in \mathscr{R}_{t}$, 


$$
\left(R_{t, 0}(A) \phi\right)(x)=\left(\begin{array}{c}
\delta_{\xi_{x, t}}(A) \phi_{1}\left(\xi_{x, t}(0)\right) \\
\delta_{\gamma_{x, t}}(A) \theta\left(\gamma_{x, t}(0)\right) \phi_{2}\left(\gamma_{x, t}(0)\right)
\end{array}\right), \quad \text { for almost all } x>0 .
$$

If $0 \leq x \leq t$, then

$$
\left(R_{t, 0}(A) \phi\right)(x)=\left(\begin{array}{c}
\delta_{\xi_{x, t}}(A) \phi_{1}\left(\xi_{x, t}(0)\right) \\
0
\end{array}\right)
$$

Consequently, $R_{t, 0}$ is supported by the set $\Omega_{t, 0}=\left\{\xi_{x, t}: x \geq 0\right\} \cup\left\{\gamma_{x, t}: x \geq t\right\}$. The two point set $\Gamma_{t, 0}=\left\{\xi_{0, t}, \gamma_{t, t}\right\}$ is the set of all paths $\omega \in \Omega_{t, 0}$ which hit the origin in the interval $[0, t]$. The set $\Gamma_{t, 0}$ clearly has $R_{t, 0}$-measure zero.

For $J=1$, there are $n+1$ terms in the sum defining $R_{t, J}(E)$ in (4.4). The first term is

$$
\begin{aligned}
V_{1}(t & \left.-t_{n}\right) Q\left(B_{n}\right) V_{0}\left(t_{n}-t_{n-1}\right) \cdots V_{0}\left(t_{2}-t_{1}\right) Q\left(B_{1}\right) V_{0}\left(t_{1}\right) \\
& =\int_{0}^{t-t_{n}} e^{T\left(t-t_{n}-\alpha\right)} U e^{T \alpha} Q\left(B_{n}\right) e^{T\left(t_{n}-t_{n-1}\right)} \cdots e^{T\left(t_{2}-t_{1}\right)} Q\left(B_{1}\right) e^{T t_{1}} d \alpha \\
& =\int_{t_{n}}^{t} e^{T(t-\alpha)} U e^{T\left(\alpha-t_{n}\right)} Q\left(B_{n}\right) e^{T\left(t_{n}-t_{n}-1\right)} \cdots e^{T\left(t_{2}-t_{1}\right)} Q\left(B_{1}\right) e^{T t_{1}} d \alpha
\end{aligned}
$$

The integrand of (4.7) is given by

$$
\begin{aligned}
& e^{T(t-\alpha)} U e^{T\left(\alpha-t_{n}\right)} Q\left(B_{n}\right) e^{T\left(t_{n}-t_{n}-1\right)} \cdots e^{T\left(t_{2}-t_{1}\right)} Q\left(B_{1}\right) e^{T t_{1}} \phi= \\
& e^{T(t-\alpha)}\left(\begin{array}{ll}
u_{11} & u_{12} \\
u_{21} & u_{22}
\end{array}\right)\left(\begin{array}{c}
\chi_{B_{n}}\left(\mathbf{x}+\alpha-t_{n}\right) \cdots \chi_{B_{1}}\left(\mathbf{x}+\alpha-t_{1}\right) \phi_{1}(\mathbf{x}+\alpha) \\
\chi_{B_{n}}\left(\mathbf{x}-\alpha+t_{n}\right) \cdots \chi_{B_{1}}\left(\mathbf{x}-\alpha+t_{1}\right) \theta(\mathbf{x}-\alpha) \phi_{2}(\mathbf{x}-\alpha)
\end{array}\right) \\
& \quad=\left(\begin{array}{l}
u_{11} \psi_{11}+u_{12} \psi_{12} \\
u_{21} \psi_{21}+u_{22} \psi_{22}
\end{array}\right)
\end{aligned}
$$

where

$$
\begin{aligned}
\begin{aligned}
\psi_{11}= & \chi_{B_{n}}\left(\mathbf{x}+t-t_{n}\right) \cdots \chi_{B_{1}}\left(\mathbf{x}+t-t_{1}\right) \phi_{1}(\mathbf{x}+t) \\
\psi_{12}= & \chi_{B_{n}}\left(\mathbf{x}+(t-\alpha)-\left(\alpha-t_{n}\right)\right) \cdots \chi_{B_{1}}\left(\mathbf{x}+(t-\alpha)-\left(\alpha-t_{1}\right)\right) \\
& \theta(\mathbf{x}+(t-\alpha)-\alpha) \phi_{2}(\mathbf{x}+(t-\alpha)-\alpha)
\end{aligned} \\
\begin{aligned}
\psi_{21}= & \chi_{B_{n}}\left(\mathbf{x}-(t-\alpha)+\alpha-t_{n}\right) \cdots \chi_{B_{1}}\left(\mathbf{x}-(t-\alpha)+\alpha-t_{1}\right) \times \\
& \theta(\mathbf{x}-(t-\alpha)) \phi_{1}(\mathbf{x}-(t-\alpha)+\alpha)
\end{aligned} \\
\begin{aligned}
\psi_{22}= & \left.\chi_{B_{n}}\left(\mathbf{x}-\left(t-t_{n}\right)\right) \cdots \chi_{B_{1}}\left(\mathbf{x}-\left(t-t_{1}\right)\right) \theta(\mathbf{x}-t)\right) \phi_{2}(\mathbf{x}-t) .
\end{aligned}
\end{aligned}
$$

If we write $\psi_{11}$ in terms of the paths $\xi_{\mathbf{x}, t}$, we get

$$
\psi_{11}(x)=\chi_{B_{n}}\left(\xi_{x, t}\left(t_{n}\right)\right) \cdots \chi_{B_{1}}\left(\xi_{x, t}\left(t_{1}\right)\right) \phi_{1}\left(\xi_{x, t}(0)\right)=\delta_{\xi_{x, t}}(E) \phi_{1}\left(\xi_{x, t}(0)\right),
$$

and for $\psi_{22}$ : 


$$
\begin{aligned}
\psi_{22}(x) & =\chi_{B_{n}}\left(\gamma_{x, t}\left(t_{n}\right)\right) \cdots \chi_{B_{1}}\left(\gamma_{x, t}\left(t_{1}\right)\right) \theta\left(\gamma_{x, t}(0)\right) \phi_{1}\left(\gamma_{x, t}(0)\right) \\
& =\delta_{\gamma_{x, t}}(E) \theta\left(\gamma_{x, t}(0)\right) \phi_{2}\left(\gamma_{x, t}(0)\right) .
\end{aligned}
$$

We require a similar expression for the terms $\psi_{12}$ and $\psi_{21}$. To do this, we introduce new paths belonging to $\Omega_{t}$ which have a change in direction at $0<\alpha<t$.

Let $\gamma_{x, t}(\alpha, \cdot)$ be the continuous path with $\gamma_{x, t}(\alpha, t)=x, \gamma_{x, t}^{\prime}(\alpha, s)=-1$ for all $\alpha<s<t$ and $\gamma_{x, t}^{\prime}(\alpha, s)=1$ for $0<s<\alpha$, so that $\gamma_{x, t}(\alpha, \cdot)$ has a change of direction at $\alpha$. Then $\gamma_{x, t}(\alpha, s)=x+(t-\alpha)-(s-\alpha)$ for all $\alpha \leq s \leq t$ and $\gamma_{x, t}(\alpha, s)=x+(t-\alpha)-(\alpha-s)$ for all $0 \leq s \leq \alpha$. Then because $0<t_{1}<\cdots<$ $t_{n} \leq \alpha \leq t$

$$
\begin{aligned}
\psi_{12}(x) & =\chi_{B_{n}}\left(\gamma_{x, t}\left(\alpha, t_{n}\right)\right) \cdots \chi_{B_{1}}\left(\gamma_{x, t}\left(\alpha, t_{1}\right)\right) \theta\left(\gamma_{x, t}(\alpha, 0)\right) \phi_{2}\left(\gamma_{x, t}(\alpha, 0)\right) \\
& =\delta_{\gamma_{x, t}(\alpha, \cdot)}(E) \theta\left(\gamma_{x, t}(\alpha, 0)\right) \phi_{2}\left(\gamma_{x, t}(\alpha, 0)\right) .
\end{aligned}
$$

Similarly, let $\xi_{x, t}(\alpha, \cdot)$ be the continuous path with $\xi_{x, t}(\alpha, t)=x, \xi_{x, t}^{\prime}(\alpha, s)=1$ for all $\alpha<s<t$ and $\xi_{x, t}^{\prime}(\alpha, s)=-1$ for $0<s<\alpha$, so that $\xi_{x, t}(\alpha, \cdot)$ has a change of direction at $\alpha$. Then $\xi_{x, t}(\alpha, s)=x-(t-\alpha)+(s-\alpha)$ for all $\alpha \leq s \leq t$ and $\xi_{x, t}(\alpha, s)=x-(t-\alpha)+(\alpha-s)$ for all $0 \leq s \leq \alpha$, and we have

$$
\begin{aligned}
\psi_{21}(x) & =\chi_{B_{n}}\left(\xi_{x, t}\left(\alpha, t_{n}\right)\right) \cdots \chi_{B_{1}}\left(\xi_{x, t}\left(\alpha, t_{1}\right)\right) \theta\left(\xi_{x, t}(\alpha, \alpha)\right) \phi_{1}\left(\xi_{x, t}(\alpha, 0)\right) \\
& =\delta_{\xi_{x, t}(\alpha, \cdot)}(E) \theta\left(\xi_{x, t}(\alpha, \alpha)\right) \phi_{1}\left(\xi_{x, t}(\alpha, 0)\right) .
\end{aligned}
$$

Thus, for almost all $x \in \mathbb{R}_{+}$, the equality

$$
\begin{aligned}
& \left(e^{T(t-\alpha)} U e^{T\left(\alpha-t_{n}\right)} Q\left(B_{n}\right) e^{T\left(t_{n}-t_{n}-1\right)} \cdots e^{T\left(t_{2}-t_{1}\right)} Q\left(B_{1}\right) e^{T t_{1}} \phi\right)(x) \\
& \quad=\left(\begin{array}{c}
u_{11} \delta_{\xi_{x, t}}(E) \phi_{1}\left(\xi_{x, t}(0)\right)+u_{12} \delta_{\gamma_{x, t}(\alpha, \cdot)}(E) \theta\left(\gamma_{x, t}(\alpha, 0)\right) \phi_{2}\left(\gamma_{x, t}(\alpha, 0)\right) \\
u_{21} \delta_{\xi_{x, t}(\alpha, \cdot)}(E) \theta\left(\xi_{x, t}(\alpha, \alpha)\right) \phi_{1}\left(\xi_{x, t}(\alpha, 0)\right)+u_{22} \delta_{\gamma_{x, t}}(E) \theta\left(\gamma_{x, t}(0)\right) \phi_{2}\left(\gamma_{x, t}(0)\right)
\end{array}\right)
\end{aligned}
$$

holds. Applying (4.7) to $\phi$ gives

$$
\begin{aligned}
& \left(\int_{t_{n}}^{t} e^{T(t-\alpha)} U e^{T\left(\alpha-t_{n}\right)} Q\left(B_{n}\right) e^{T\left(t_{n}-t_{n}-1\right)} \cdots e^{T\left(t_{2}-t_{1}\right)} Q\left(B_{1}\right) e^{T t_{1}} \phi d \alpha\right)(x) \\
& =u_{11} \delta_{\xi_{x, t}}(E)\left(\begin{array}{c}
\phi_{1}\left(\xi_{x, t}(0)\right) \\
0
\end{array}\right)\left(t-t_{n}\right) \\
& \quad+u_{12} \int_{t_{n}}^{t} \delta_{\gamma_{x, t}(\alpha, \cdot)}(E)\left(\begin{array}{c}
\theta\left(\gamma_{x, t}(\alpha, 0)\right) \phi_{2}\left(\gamma_{x, t}(\alpha, 0)\right) \\
0
\end{array}\right) d \alpha \\
& \quad+u_{21} \int_{t_{n}}^{t} \delta_{\xi_{x, t}(\alpha, \cdot)}(E)\left(\begin{array}{c}
0 \\
\theta\left(\xi_{x, t}(\alpha, \alpha)\right) \phi_{1}\left(\xi_{x, t}(\alpha, 0)\right.
\end{array}\right) d \alpha \\
& 0 \\
& \quad+u_{22} \delta_{\gamma_{x, t}}(E)\left(\begin{array}{c}
0 \\
\theta\left(\gamma_{x, t}(0)\right) \phi_{2}\left(\gamma_{x, t}(0)\right)
\end{array}\right)\left(t-t_{n}\right) .
\end{aligned}
$$


Adding the other $n$ expressions in (4.4) yields the equality

$$
\begin{aligned}
\left(R_{t, 1}(E) \phi\right)(x)= & u_{11} \delta_{\xi_{x, t}}(E)\left(\begin{array}{c}
\phi_{1}\left(\xi_{x, t}(0)\right) \\
0
\end{array}\right) t \\
& +u_{12} \int_{0}^{t} \delta_{\gamma_{x, t}(\alpha, \cdot)}(E)\left(\begin{array}{c}
\theta\left(\gamma_{x, t}(\alpha, 0)\right) \phi_{2}\left(\gamma_{x, t}(\alpha, 0)\right) \\
0
\end{array}\right) d \alpha \\
& +u_{21} \int_{0}^{t} \delta_{\xi_{x, t}(\alpha, \cdot)}(E)\left(\begin{array}{c}
0 \\
\theta\left(\xi_{x, t}(\alpha, \alpha)\right) \phi_{1}\left(\xi_{x, t}(\alpha, 0)\right.
\end{array}\right) d \alpha \\
& +u_{22} \delta_{\gamma_{x, t}}(E)\left(\begin{array}{c}
0 \\
\theta\left(\gamma_{x, t}(0)\right) \phi_{2}\left(\gamma_{x, t}(0)\right)
\end{array}\right) t
\end{aligned}
$$

a representation which is indepencent of the form of the elementary event $E$. The function $\gamma_{x, t}(\alpha, \cdot)$ takes its minimum value $\min \left(x, \gamma_{x, t}(\alpha, 0)\right)$ at one of the endpoints of the time interval $[0, t]$. The minimum value of $\xi_{x, t}(\alpha, \cdot)$ is $\left.\xi_{x, t}(\alpha, \alpha)\right)$.

Let $\left\{\gamma_{x, t}(\alpha, \cdot) \geq 0\right\}$ denote the set of all $0 \leq \alpha \leq t$ for which the path $\gamma_{x, t}(\alpha, \cdot)$ belongs to $\Omega_{t}$. Similarly for $\left\{\xi_{x, t}(\alpha, \cdot) \geq 0\right\}$. We have derived the following expression for $R_{t, 1}$ :

Proposition 4.2. For every $A \in \mathscr{R}_{t}$, we have

$$
\begin{aligned}
& \left(R_{t, 1}(A) \phi\right)(x)=u_{11} \delta_{\xi_{x, t}}(A)\left(\begin{array}{c}
\phi_{1}\left(\xi_{x, t}(0)\right) \\
0
\end{array}\right) t \\
& +u_{12} \int_{\left\{\gamma_{x, t}(\alpha, \cdot) \geq 0\right\}} \delta_{\gamma_{x, t}(\alpha, \cdot)}(A)\left(\begin{array}{c}
\phi_{2}\left(\gamma_{x, t}(\alpha, 0)\right) \\
0
\end{array}\right) d \alpha \\
& +u_{21} \int_{\left\{\xi_{x, t}(\alpha, \cdot) \geq 0\right\}} \delta_{\xi_{x, t}(\alpha, \cdot)}(A)\left(\begin{array}{c}
0 \\
\phi_{1}\left(\xi_{x, t}(\alpha, 0)\right.
\end{array}\right) d \alpha \\
& +u_{22} \delta_{\gamma_{x, t}}(A)\left(\begin{array}{c}
0 \\
\theta\left(\gamma_{x, t}(0)\right) \phi_{2}\left(\gamma_{x, t}(0)\right)
\end{array}\right) t .
\end{aligned}
$$

It follows that $R_{t, 1}$ is concentrated on the set $\Omega_{t, 1}$ of paths $\omega \in \Omega_{t}$ with at most one change of direction in the interval $[0, t]$. The set $\Xi_{t, 1}$ of paths $\omega \in \Omega_{t, 1}$ which hit the origin in the interval $[0, t]$ is the set

$$
\left\{\xi_{0, t}, \gamma_{t, t}\right\} \cup\left\{\xi_{x, t}(t-x, \cdot), \gamma_{x, t}((x+t) / 2, \cdot): 0 \leq x \leq t\right\} \cup\left\{\gamma_{0, t}(\alpha, \cdot): 0 \leq \alpha \leq t\right\},
$$

compact for the topology on $\Omega_{t}$ of uniform convergence on the interval $[0, t]$.

On appealing to Fubini's theorem, we see that for any subset $A \in \mathscr{R}_{t}$ contained in $\Gamma_{t, 1}$, the integral $\int_{\mathbb{R}_{+}}\left|\left(R_{t, 1}(A) \phi\right)(x)\right| d x$ is zero. Therefore, $\Gamma_{t, 1}$ is an $R_{t, 1}$-null set.

Having computed the cases $J=0,1$ explicitly, we show that there exists 
an analogous representation in the cases $J=2,3, \ldots$, although we shall not derive an exact formula in general. The main features are already apparent for $J=1$.

The terms in the sum (4.4) can be written as

$$
\begin{aligned}
V_{j_{n}}\left(t-t_{n}\right) Q\left(B_{n}\right) V_{j_{n-1}}\left(t_{n}-t_{n-1}\right) \cdots V_{j_{1}}\left(t_{2}-t_{1}\right) Q\left(B_{1}\right) V_{j_{0}}\left(t_{1}\right)= \\
\quad \int_{W} P_{n}\left(\alpha_{j_{0}}+\cdots+j_{n-1}+1, \ldots, \alpha_{j_{0}+\cdots+j_{n}}\right) \times \\
\prod_{k=0}^{n-1}\left[Q\left(B_{k+1}\right) P_{k}\left(\alpha_{j_{0}+\cdots+j_{k-1}+1}, \ldots, \alpha_{j_{0}+\cdots+j_{k}}\right)\right] d \alpha_{1} \cdots d \alpha_{J}
\end{aligned}
$$

If $j_{k} \neq 0$, the operator valued function $P_{k}\left(\alpha_{j_{0}}+\cdots+j_{k-1}+1, \ldots, \alpha_{j_{0}}+\cdots+j_{k}\right)$ is equal to

$$
\begin{aligned}
& e^{T\left(t_{k+1}-\alpha_{j 0}+{ }^{\prime}\right)} U e^{T\left(\alpha_{j 0}+\cdots+J_{k}-\alpha_{j 0}+\cdot+j_{k}-1\right)} \ldots \\
& \cdots e^{T\left(\alpha_{J 0}+J_{k-1}+2-\alpha_{J 0^{+}}+J_{k-1}+1\right)} U e^{T\left(\alpha_{J 0}+\cdot+J_{k-1}+1-t_{k}\right)},
\end{aligned}
$$

otherwise it is equal to the constant operator $\exp \left(T\left(t_{k+1}-t_{k}\right)\right)$. The set $W$ is the ordered set of $J$ points in the interval $[0, t]$ such that there are exactly $j_{k}$ points in the intervals $\left[t_{k}, t_{k+1}\right]$, where $k=0, \ldots, n, t_{0}=0$ and $t_{n+1}=t$.

The integrand in (4.10) can be expressed as a finite linear combination of functions

$$
\begin{aligned}
& Z_{n}\left(\alpha_{j_{0}+\cdots+j_{n-1}}, \ldots, \alpha_{j_{0}+\cdots+j_{n}}\right) \times \\
& \quad \prod_{k=1}^{n-1}\left[Q\left(B_{k+1}\right) Z_{k}\left(\alpha_{j_{0}+\cdots+j_{k-1}+1}, \ldots, \alpha_{j_{0}}+\cdots+j_{k}\right)\right] Q\left(B_{1}\right) Z_{0}\left(\alpha_{1}, \ldots, \alpha_{j_{0}}\right) .
\end{aligned}
$$

If $j_{k} \neq 0$, the operator valued function $Z_{k}\left(\alpha_{j_{0}+\cdots+j_{k-1}+1}, \ldots, \alpha_{j_{0}+\cdots+j_{k}}\right)$ is equal to

$$
\begin{aligned}
& \left.e^{T\left(t_{k+1}-\alpha_{j 0+}+{ }^{\prime}\right)} E_{j_{0}+\cdots+j_{k}} e^{T\left(\alpha_{j 0}+\right.}+{ }_{j k}-\alpha_{j 0}+\quad+J_{k}-1\right) \ldots \\
& \cdots e^{T\left(\alpha_{j 0}+j_{k-1}+2-\alpha_{j 0}++J_{k-1}+1\right)} E_{j_{0}+\cdots+j_{k-1}+1} e^{T\left(\alpha_{j 0+}+J_{k-1}+1-t_{k}\right)},
\end{aligned}
$$

for some choice of matrices $E_{k}, k=1, \ldots, J$ from the standard basis $\mathscr{E}$ of the $2 \times 2$ matrices. If $j_{k}=0$, then it is equal to the constant operator $\exp \left(T\left(t_{k+1}-t_{k}\right)\right)$.

Let $\beta:\{1, \ldots, J\} \rightarrow\{0,1\}$ and $0<\alpha_{1}<\cdots<\alpha_{J}<t . \quad$ Let $\gamma_{x, t}^{+}\left(\alpha_{1}, \ldots, \alpha_{J}, \beta, \cdot\right)$ (respectively, $\gamma_{x, t}^{-}\left(\alpha_{1}, \ldots, \alpha_{J}, \beta, \cdot\right)$ ) denote the path $\omega:[0, t] \rightarrow \mathbb{R}$, such that $\omega(t)=x, \omega^{\prime}(t)=1$ (respectively, $\omega^{\prime}(t)=-1$ ) and for each $j=1, \ldots, J$, there is a change in direction of $\omega$ at $\alpha_{j}$ if and only if $\beta(j)=1$; elsewhere, $\omega$ has velocity \pm 1 . In terms of the paths employed in Proposition 4.2 for the case $J=1$, we have $\gamma_{x, t}=\gamma_{x, t}^{+}(\alpha, 0, \cdot), \xi_{x, t}=\gamma_{x, t}^{-}(\alpha, 0, \cdot), \gamma_{x, t}(\alpha, \cdot)=\gamma_{x, t}^{-}(\alpha, 1, \cdot)$, and $\xi_{x, t}(\alpha, \cdot)=\gamma_{x, t}^{+}(\alpha, 1, \cdot)$.

We shall use the notation $\gamma_{\mathbf{x}, t}^{ \pm}\left(\alpha_{1}, \ldots, \alpha_{J}, \beta, \cdot\right)$ to denote the function which assigns to each point $x \in \mathbb{R}_{+}$the path $\gamma_{x, t}^{ \pm}\left(\alpha_{1}, \ldots, \alpha_{J}, \beta, \cdot\right)$. We use the 
terminology path function here.

Lemma 4.3. Let $\phi \in L^{2}\left(\mathbb{R}_{+} ; \mathbb{C}^{2}\right)$. Then there exists a map $\beta:\{1, \ldots, J\} \rightarrow$ $\{0,1\}$, a path function $\omega_{\mathbf{x}}^{ \pm}=\gamma_{\mathbf{x}, t}^{ \pm}\left(\alpha_{1}, \ldots, \alpha_{J}, \beta, \cdot\right)$ taking values in $\Omega_{t}$, and $a$ matrix $M \in \mathscr{E} \cup\{0\}$, such that for every elementary event $E \subseteq \Omega_{t}$ of the form (1.1), the equality

$$
\begin{aligned}
& Z_{n}\left(\alpha_{j_{0}+\cdots+j_{n-1}+1}, \ldots, \alpha_{j_{0}+\cdots+j_{n}}\right) \times \\
& \quad\left[\prod_{k=1}^{n-1} Q\left(B_{k+1}\right) Z_{k}\left(\alpha_{j_{0}+\cdots+j_{k-1}+1}, \ldots, \alpha_{j_{0}+\cdots+j_{k}}\right)\right] Z_{0}\left(\alpha_{1}, \ldots, \alpha_{j_{0}}\right) \phi \\
& =\delta_{\omega_{\mathbf{x}}^{ \pm}}(E)(M \phi)\left(\omega_{\mathbf{x}}^{ \pm}(0)\right),
\end{aligned}
$$

holds almost everywhere. If $E_{J}=\varepsilon_{1}$ or $E_{J}=\varepsilon_{2}$, then the path function $\omega_{\mathbf{x}}^{-}$ appears, otherwise, it is $\omega_{\mathbf{x}}^{+}$.

Proof. Let

$$
\varepsilon_{1}=\left(\begin{array}{ll}
1 & 0 \\
0 & 0
\end{array}\right), \quad \varepsilon_{2}=\left(\begin{array}{ll}
0 & 1 \\
0 & 0
\end{array}\right), \quad \varepsilon_{3}=\left(\begin{array}{ll}
0 & 0 \\
1 & 0
\end{array}\right), \quad \varepsilon_{4}=\left(\begin{array}{ll}
0 & 0 \\
0 & 1
\end{array}\right)
$$

be the standard basis elements of the $2 \times 2$ matrices.

The action of the operator $e^{T s}$ on $\psi \in L^{2}\left(\mathbb{R}_{+} ; \mathbb{C}^{2}\right)$ is to translate the graph of the first component $\psi_{1}$ of $\psi$ to the left by an amount $s$, and the graph of the second component $\psi_{2}$ to the right by an amount $s$, setting $\left(e^{T s} \psi\right)_{2}(x)$ equal to the zero for $0 \leq x \leq s$. However, on encountering a basis element $\varepsilon_{2}$ or $\varepsilon_{3}, e^{T s}$ translates a component in the opposite direction. For example, $\left(e^{T s} \varepsilon_{2} \psi\right)_{2}=0$ and the graph of $\left(e^{T s} \varepsilon_{2} \psi\right)_{1}$ is the translate of $\psi_{2}$ to the left by an amount $s$.

It is clear that the left hand side of (4.12) is some translate of the function $M \phi$, times a scalar function which takes the values zero or one, and depending on the sets $B_{1}, \ldots, B_{n}$. Here the matrix $M$ is the product $E_{J} \cdots E_{1}$ of the basis matrices appearing in (4.11).

If the matrix $M$ is the zero matrix, then $\beta$ and the path function $\omega_{\mathbf{x}}^{ \pm}$ can be chosen arbitrarily - both sides of (4.12) are identically zero.

Suppose now that $M \neq 0$. For each $j=1, \ldots, J$, set $\beta(j)=1$ if $E_{j}$ is equal to either $\varepsilon_{2}$ or $\varepsilon_{3}$, and set $\beta(j)=0$ otherwise. Nonzero values of the function $\beta$ correspond to changes in direction at the corresponding times $\alpha_{j}$. We shall prove formula (4.12) by induction on $J$. The case $J=1$ was established for all $t>0$ in the course of proving Proposition 4.2.

Suppose that for $J=K$, formula (4.12) is true with the choice above for the path function $\omega_{\mathrm{x}}^{ \pm}$and the matrix $M \neq 0$, for all $t>0$, all elementary events of the form (1.1), and all choices of non-negative integers $j_{0}, \ldots, j_{n}$ such that $j_{0}+\cdots+j_{n}=K$, and $n=1,2, \ldots$ Let $j_{0}, \ldots, j_{n}$ be nonnegative integers such that $j_{0}+\cdots+j_{n}=K+1$. Suppose first that $j_{n}>0$. 
The expression (4.11) for $Z_{n}\left(\alpha_{j_{0}+\cdots+j_{n-1}+1}, \ldots, \alpha_{K+1}\right)$ can be written as

$$
\exp \left(T\left(t-\alpha_{K+1}\right)\right) E_{K+1} Z_{n}\left(\alpha_{j_{0}+\cdots+j_{n-1}+1}, \ldots, \alpha_{K}\right)
$$

with the term $Z_{n}$ here defined by the expression (4.11) with $t_{k+1}=\alpha_{K+1}$. Let $\psi$ be the second factor on the left-hand side of equation (4.12). By the induction hypothesis,

$$
Z_{n}\left(\alpha_{j_{0}+\cdots+j_{n-1}+1}, \ldots, \alpha_{K}\right) \psi=\delta_{\omega_{\mathbf{x}}}(E)(M \phi)\left(\omega_{\mathbf{x}}(0)\right),
$$

for the path function $\omega_{\mathbf{x}}$ and matrix $M$ defined above with the choice $t=\alpha_{K+1}$. Then

$$
Z_{n}\left(\alpha_{j_{0}+\cdots+j_{n-1}+1}, \ldots, \alpha_{K+1}\right) \psi=\exp \left(T\left(t-\alpha_{K+1}\right)\right) \delta_{\omega_{\mathbf{x}}}(E)\left(E_{K+1} M \phi\right)\left(\omega_{\mathbf{x}}(0)\right) .
$$

Only the case $E_{K+1} M \neq 0$ is of interest. We examine the possible choices of $E_{K+1} \in \mathscr{E}$.

If $E_{K+1}$ is either $\varepsilon_{1}$ or $\varepsilon_{4}$, then $E_{K+1} M=M$. In the case that $E_{K+1}=\varepsilon_{1}$, then we can only have $E_{K}=\varepsilon_{1}$ and $\beta(K)=0$, or $E_{K}=\varepsilon_{2}$ and $\beta(K)=1$, otherwise $E_{K+1} M$ would be the zero matrix. In the either case,

$$
Z_{n}\left(\alpha_{j_{0}+\cdots+j_{n-1}+1}, \ldots, \alpha_{K+1}\right) \psi=\delta_{\omega_{\mathbf{x}+\left(t-\alpha_{K}+1\right)}}(E)(M \phi)\left(\omega_{\mathbf{x}+\left(t-\alpha_{K+1}\right)}(0)\right) .
$$

By the induction hypothesis, the path $\omega_{x} \in \Omega_{\alpha_{K+1}}$ is equal to

$$
\gamma_{x, \alpha_{K}+1}^{-}\left(\alpha_{1}, \ldots, \alpha_{K}, \beta, \cdot\right),
$$

for each $x \in \mathbb{R}_{+}$, because $E_{K}$ is equal to $\varepsilon_{1}$ or $\varepsilon_{2}$. Then $\omega_{x+\left(t-\alpha_{K}+1\right)}$ is the path $\tilde{\omega}_{x}=\gamma_{x, t}^{-}\left(\alpha_{1}, \ldots, \alpha_{K+1}, \tilde{\beta}, \cdot\right)$ with the function $\tilde{\beta}:\{1, \ldots, K+1\} \rightarrow\{0,1\}$ defined by $\tilde{\beta}(j)=\beta(j)$ for all $j=1, \ldots, K$ and $\tilde{\beta}(K+1)=0$ and $\tilde{\omega}_{x}$ has derivative -1 in the open interval $\left(\alpha_{K}, t\right)$. Thus,

$$
Z_{n}\left(\alpha_{j_{0}+\cdots+j_{n-1}+1}, \ldots, \alpha_{K+1}\right) \psi=\delta_{\tilde{\omega}_{\mathbf{x}}}(E)(M \psi)\left(\tilde{\omega}_{\mathbf{x}}(0)\right) .
$$

The negative sign appears in the path function $\tilde{\omega}_{\mathbf{x}}$ in the case $E_{K+1}=\varepsilon_{1}$.

In the case $E_{K+1}=\varepsilon_{4}$, we obtain

$$
\begin{aligned}
& Z_{n}\left(\alpha_{j_{0}+\cdots+j_{n-1}+1}, \ldots, \alpha_{K+1}\right) \psi= \\
& \quad \theta\left(\mathbf{x}-\left(t-\alpha_{K+1}\right)\right) \delta_{\omega_{\mathbf{x}-\left(t-\alpha_{K}+1\right)}}(E)(M \psi)\left(\omega_{\mathbf{x}-\left(t-\alpha_{K}+1\right)}(0)\right) .
\end{aligned}
$$

By the induction hypothesis, the path $\omega_{x} \in \Omega_{\alpha_{K+1}}$ is equal to

$$
\gamma_{x, \alpha_{K}+1}^{+}\left(\alpha_{1}, \ldots, \alpha_{K}, \beta, \cdot\right),
$$

for each $x \in \mathbb{R}_{+}$, because $E_{K}$ is equal to $\varepsilon_{2}$ or $\varepsilon_{4}$. Then $\omega_{x-\left(t-\alpha_{K+1}\right)}$ is the path $\tilde{\omega}_{x}=\gamma_{x, t}^{+}\left(\alpha_{1}, \ldots, \alpha_{K+1}, \tilde{\beta}, \cdot\right)$ with the function $\tilde{\beta}:\{1, \ldots, K+1\} \rightarrow\{0,1\}$ defined by $\tilde{\beta}(j)=\beta(j)$ for all $j=1, \ldots, K$ and $\tilde{\beta}(K+1)=0$ and $\tilde{\omega}_{x}$ has derivative +1 in the open interval $\left(\alpha_{K}, t\right)$. If $x<t-\alpha_{K+1}$, then $\tilde{\omega}_{x}$ does not belong to the space $\Omega_{t}$ of paths taking values in $\mathbb{R}_{+}$, so $\delta_{\tilde{\omega}_{\mathbf{x}}}(E)=0$. Thus, 


$$
Z_{n}\left(\alpha_{j_{0}}+\cdots+j_{n-1}+1, \ldots, \alpha_{K+1}\right) \psi=\delta_{\tilde{\omega}_{\mathbf{x}}}(E)(M \psi)\left(\tilde{\omega}_{\mathbf{x}}(0)\right) .
$$

The positive sign appears in the path function $\tilde{\omega}_{\mathbf{x}}$ in the case $E_{K+1}=\varepsilon_{4}$.

The other cases $E_{K+1}=\varepsilon_{2}$ and $E_{K+1}=\varepsilon_{3}$ correspond to changes in direction of the path $\omega_{x}, x \in \mathbb{R}_{+}$, at time $\alpha_{K+1}$ with $\alpha_{K}<\alpha_{K+1}<t$. The verification of the induction hypothesis for $J=K+1$ is similar to that above.

It remains to treat the case $j_{n}=0$. For some $m=0, \ldots, n-1$, we have $j_{m}>0$ and $j_{0}+\cdots+j_{m}=K+1$. Let

$$
\psi=\left[\prod_{k=1}^{m} Q\left(B_{k+1}\right) Z_{k}\left(\alpha_{j_{0}+\cdots+j_{k-1}+1}, \ldots, \alpha_{j_{0}+\cdots+j_{k}}\right)\right] Z_{0}\left(\alpha_{1}, \ldots, \alpha_{j_{0}}\right) \phi .
$$

Then, under the induction hypothesis, we have established that

$$
\psi=\delta_{\omega_{\mathbf{x}}^{ \pm}}(A)[M \phi]\left(\omega_{\mathbf{x}}^{ \pm}(0)\right)
$$

for the matrix $M=E_{K+1} \cdots E_{1}$. If $E_{K+1}=\varepsilon_{1}$ or $E_{K+1}=\varepsilon_{2}$, then $\omega_{\mathbf{x}}^{-} \in \Omega_{t_{m+1}}$ appears, otherwise, it is $\omega_{\mathbf{x}}^{+} \in \Omega_{t_{m+1}}$. As the argument above shows, an application of the operator

$$
e^{T\left(t-t_{n}\right)} Q\left(B_{n}\right) \cdots e^{T\left(t_{m}+3-t_{m}+2\right)} Q\left(B_{m+2}\right) e^{T\left(t_{m}+2-t_{m}+1\right)}
$$

to $\psi$ shows that (4.12) is also true in the case $j_{n}=0$, so the result follows by induction for all $J=1,2, \ldots$.

The following statement is a generalisation of equation (4.9) to the case $J>1$. Given $x \geq 0, t>0$ and a map $\beta:\{1, \ldots, J\} \rightarrow\{0,1\}$, let $\left\{\gamma_{x, t}^{ \pm}\left(\alpha_{1}, \ldots\right.\right.$, $\left.\left.\alpha_{J}, \beta, \cdot\right) \geq 0\right\}$ denote the set of all $J$-tuples $0<\alpha_{1}<\cdots<\alpha_{J}<t$ belonging to $[0, t]$ for which the path $\gamma_{x, t}^{ \pm}\left(\alpha_{1}, \ldots, \alpha_{J}, \beta, \cdot\right)$ belongs to $\Omega_{t}$, that is, where it has its values in $\mathbb{R}_{+}$. The set $\left\{\gamma_{x, t}^{ \pm}\left(\alpha_{1}, \ldots, \alpha_{J}, \beta, \cdot\right) \geq 0\right\}$ is possibly empty.

Proposition 4.4. Let $\phi \in L^{2}\left(\mathbb{R}_{+} ; \mathbb{C}^{2}\right)$. The measure $R_{t, J} \phi$ is a finite linear combination of $L^{2}\left(\mathbb{R}_{+} ; \mathbb{C}^{2}\right)$-valued measures

$$
\begin{aligned}
A \mapsto & \int_{\left\{\gamma_{\mathbf{x}, t}^{ \pm}\left(\alpha_{1}, \ldots, \alpha_{J}, \beta, \cdot\right) \geq 0\right\}} \delta_{\gamma_{\mathbf{x}, t}^{ \pm}\left(\alpha_{1}, \ldots, \alpha_{J}, \beta, \cdot\right)}(A) \times \\
& (M \phi)\left(\gamma_{\mathbf{x}, t}^{ \pm}\left(\alpha_{1}, \ldots, \alpha_{J}, \beta, 0\right)\right) d \alpha_{1} \cdots d \alpha_{J}, \quad A \in \mathscr{R}_{t},
\end{aligned}
$$

for some maps $\beta:\{1, \ldots, J\} \rightarrow\{0,1\}$, path functions $\gamma_{\mathbf{x}, t}^{ \pm}\left(\alpha_{1}, \ldots, \alpha_{J}, \beta, \cdot\right)$ and matrices $M \in \mathscr{E}$. In this notation, the element of $L^{2}\left(\mathbb{R}_{+} ; \mathbb{C}^{2}\right)$ on the right-hand side of (4.13) is the function which assigns to each $x \in \mathbb{R}_{+}$, the element of $\mathbb{C}^{2}$ obtained by replacing $\mathbf{x}$ by $x$.

Proof. The integrand in (4.10) can be expressed as a finite linear combination of the expressions (4.12) by writing the matrix $U$ as a linear combination of matrices from the standard basis $\mathscr{E}$. The corresponding sum over the expression (4.10) for all non-negative integers $j_{0}+\cdots+j_{n}=J$ is a 
finite linear combination of integrals of the expression (4.12) over the set $0<\alpha_{1}<\cdots<\alpha_{J}<t$ in $[0, t]^{J}$.

Let $x \in \mathbb{R}_{+}, \beta:\{1, \ldots, J\} \rightarrow\{0,1\}$ and $A \in \mathscr{R}_{t}$. Suppose that at some point $0<\alpha_{1}<\cdots<\alpha_{J}<t$, the path $\gamma_{x, t}^{ \pm}\left(\alpha_{1}, \ldots, \alpha_{J}, \beta, \cdot\right)$ takes on negative values in the interval $[0, t]$. Then $\delta_{\gamma_{x, t}^{ \pm}\left(\alpha_{1}, \ldots, \alpha_{J}, \beta, \cdot\right)}(A)=0$, because $A$ is a subset of $\Omega_{t}$ whose elements assume values only in the set $\mathbb{R}_{+}$. The region of integration in which the integrand is nonzero is therefore contained in the set $\left\{\gamma_{x, t}^{ \pm}\left(\alpha_{1}, \ldots, \alpha_{J}, \beta, \cdot\right) \geq 0\right\}$.

The expression on the right hand side of (4.13) does not depend on the form of an elementary event $A$ like (1.1), and we have shown that for all elementary events $A$ of the form (1.1), the operator valued set function defined by (4.4) is equal to a finite linear combination of operator valued set functions defined by (4.13), so equality holds on the $\sigma$-algebra $\mathscr{R}_{t}$ by $\sigma$-additivity.

Let $\Gamma_{t}$ be the set of all paths $\omega \in \Omega_{t}$ which take the value zero at least once in the interval $[0, t]$. Paths belonging to $\Omega_{t}$ are continuous, so $\Gamma_{t} \in \mathscr{R}_{t}$ by virtue of the equality $\Gamma_{t}=\bigcap_{m=1}^{\infty} \bigcup_{j=1}^{\infty} \bigcap_{n=j}^{\infty} \bigcup_{k=1}^{n}\left\{\omega \in \Omega_{t}: \omega(t k / n)<1 / m\right\}$.

Corollary 4.5. The set $\Gamma_{t}$ is an $R_{t}$-null set.

Proof. The measure $R_{t}$ is the setwise sum of the measures $R_{t, J}$, $J=0,1,2 \ldots$ on the $\sigma$-algebra $\mathscr{R}_{t}$, in the strong operator topology of $\mathscr{L}\left(L^{2}\left(\mathbb{R}_{+} ; \mathbb{C}^{2}\right)\right)$, so it is enough to show that $\Gamma_{t}$ is an $R_{t, J}$-null set, for each $J=0,1, \ldots$ For $J=0,1$, we verified this by writing down the set $\Gamma_{t, J}$ explicitly. In general, for almost all $x \in \mathbb{R}_{+}$, the set

$$
\left\{\left(\alpha_{1}, \ldots, \alpha_{J}\right): 0<\alpha_{1}<\cdots<\alpha_{J}<t, 0 \in \gamma_{x, t}^{ \pm}\left(\alpha_{1}, \ldots, \alpha_{J}, \beta,[0, t]\right)\right\}
$$

is contained in the finite union of hyperplanes in $[0, t]^{J}$, so it has Lebesgue measure zero.

For any subset $A \in \mathscr{R}_{t}$ of $\Gamma_{t}$, it follows that the integral on the right hand side of expression (4.13) is zero at almost all points $x \in \mathbb{R}_{+}$, so $\mathbb{R}_{t, J}(A)=0$, proving that $\Gamma_{t}$ is $R_{t, J}$-null.

Let $\Omega_{t, J}, J=0,1, \ldots$ be the set of all paths $\omega \in \Omega_{t}$ with at most $J$ changes in direction in the interval $[0, t]$. Then $\Omega_{t, J} \subset \Omega_{t, J+1}$ and $\Omega_{t}=\bigcup_{J=0}^{\infty} \Omega_{t, J}$. The following corollary is proved in [ $\mathbb{J} 2$, Lemma 4.2] without mentioning the formula (4.13) explicitly.

Corollary 4.6. For each $J=0,1, \ldots$, the measure $\mathbb{R}_{t, J}$ is supported by $\Omega_{t, J}$.

If the the matrix $U$ is replaced by the bounded matrix valued function $i N^{*} U_{k, \varepsilon} N$, then $N^{*} R_{t}^{(k, \varepsilon)} N$ may be represented as the sum of measures like (4.13), except that the integrand is multiplied by additional functions of $\alpha_{1}, \ldots, \alpha_{J}$ and $x$ associated with the translates of the components of $i N^{*} \mathbb{U}_{k, \varepsilon} N$; in the situation considered above, these were constants. It is clear that this 
change will not affect the conclusions of Corollary 4.5 with respect to the new measure $N^{*} R_{t}^{(k, \varepsilon)} N$. We state the following without writing down a formal proof:

Proposition 4.7. The set $\Gamma_{t}$ is an $R_{t}^{(k, \varepsilon)}$-null set for all $\varepsilon>0$ and all nonzero integers $k$.

Corollary 4.8. The set $\Gamma_{t}$ is an $M_{t}^{+}$-null set.

Proof. For all $\varepsilon>0$, the operator valued measure $M_{t}^{(\varepsilon)}$ is the direct sum of measures unitarily equivalent to $R_{t}^{(k, \varepsilon)}$, for $k= \pm 1, \pm 2, \ldots$ If $A \in \mathscr{R}_{t}$ is a subset of $\Gamma_{t}$, then $R_{t}^{(k, \varepsilon)}(A)=0$ for all $k= \pm 1, \pm 2, \ldots$, so that $M_{t}^{(\varepsilon)}(A)=0$. Hence, $\Gamma_{t}$ is an $M_{t}^{+}$-null set.

\section{§5. The Feynman Representation}

In this section, we use Corollary 4.8 to complete the proof of the Feynman representation $(0.1)$ for a class of potentials $q$ which includes a range of Coulomb potentials.

Let $q: \mathbb{R}_{++} \rightarrow \mathbb{R}$ be a locally square integrable function. Let $\mathscr{D}(\tau(q))$ be the set of all functions $\phi \in L^{2}\left(\mathbb{R}_{+} ; \mathbb{C}^{2}\right)$ which are absolutely continuous on all bounded subintervals of $\mathbb{R}_{+}$, such that $\left(N^{*} \phi\right)_{2}(0)=0$ and $\tau(q) \phi:=\tau \phi-Q(q) \phi$ $\in L^{2}\left(\mathbb{R}_{+} ; \mathbb{C}^{2}\right)$. It is proved in $[\mathrm{J} 2$, Proposition 2.3] that the operator $i \tau(q)$ : $\mathscr{D}(\tau(q)) \rightarrow L^{2}\left(\mathbb{R}_{+} ; \mathbb{C}^{2}\right)$ is the infinitesimal generator of a $\mathrm{C}_{0}$-contraction semigroup $e^{i \tau(q) t}, t \geq 0$. For each $t \geq 0$, the operator $e^{i \tau(q) t}$ maps $\phi \in L^{2}\left(\mathbb{R}_{+} ; \mathbb{C}^{2}\right)$ to the function given by

$$
x \mapsto N\left(\begin{array}{c}
\exp \left[-i \int_{0}^{t} q(x+s) d s\right]\left(N^{*} \phi\right)_{1}(x+t) \\
\theta(x-t) \exp \left[-i \int_{0}^{t} \theta(x-t) q(x-s) d s\right]\left(N^{*} \phi\right)_{2}(x-t)
\end{array}\right),
$$

for almost all $x>0$; calculating the resolvent of the semigroup so defined shows that the generator is indeed $i \tau(q)$.

The function $q$ need not be integrable in a neighbourhood of zero, although it is necessarily integrable over all bounded intervals in $\mathbb{R}_{++}$. Nevertheless, the formula (5.1) makes sense for almost all $x>0$ and defines a function in $L^{2}\left(\mathbb{R}_{+} ; \mathbb{C}^{2}\right)$.

Any bounded perturbation of $\tau(q)$ is the generator of a $\mathrm{C}_{0}$-semigroup [K1, Theorem IX.2.1], so for all $\varepsilon>0$ and $k \in \mathbb{Z} \backslash\{0\}, \tau(q)+U_{k, \varepsilon}$ is the generator of a $\mathrm{C}_{0}$-semigroup. Because $U_{k, \varepsilon}(r)$ is an hermitian matrix for each $r>0$, it follows from the Trotter product formula that $\tau(q)+U_{k, \varepsilon}$ is the generator of a contraction $C_{0}$-semigroup on $L^{2}\left(\mathbb{R}_{+} ; \mathbb{C}^{2}\right)$. By the notation $\tau_{k, \varepsilon} \dot{-} q$, we mean the operator with domain $\mathscr{D}(\tau(q))$ equal to $\tau(q)+U_{k, \varepsilon}$. 
The direct sum

$$
\sum_{k, m \in K_{\varepsilon}} \oplus \mathscr{U}_{k, m}^{*}\left(\tau_{k, \varepsilon} \dot{-} q\right) \mathscr{U}_{k, m}-\sum_{|k|>1 / \varepsilon} \oplus\left(\sum_{m=-|k|}^{|k|-1} \oplus \mathscr{U}_{k, m}^{*} Q(q) \mathscr{U}_{k, m}\right)
$$

over the set $K_{\varepsilon}$ all integers $k, m$ such that $m=-|k|,-|k|+1, \ldots,|k|-1$ and $1 \leq|k| \leq 1 / \varepsilon$ is denoted by $D_{\varepsilon} \dot{-} Q_{r}(q)$. Then $i\left(D_{\varepsilon} \dot{-} Q_{r}(q)\right)$ is the direct sum of infinitesimal generators of $\mathrm{C}_{0}$-semigroups, so it is itself the infinitesimal generators of a $\mathrm{C}_{0}$-semigroup. In the case that $q$ is bounded and measurable, the operator $D_{\varepsilon}-Q_{r}(q)$ so defined is, in fact, the difference between the unbounded operator $D_{\varepsilon}$ and the bounded operator $Q_{r}(q)$, because $Q_{r}(q)=$ $\sum_{|k|=1}^{\infty} \oplus\left(\sum_{m=-|k|}^{|k|-1} \oplus \mathscr{U}_{k, m}^{*} Q(q) \mathscr{U}_{k, m}\right)$.

Lemma 5.1. Let $q: \mathbb{R}_{++} \rightarrow \mathbb{R}$ be a locally integrable function and $t>0$. Then for $M_{t}^{+}$-almost all $\omega \in \Omega$, the function $s \mapsto q(\omega(s))$ is Lebesgue integrable on $[0, t]$.

Proof. According to Corollary 4.8, the set $\Gamma_{t}$ of all paths $\omega \in \Omega$ which hit the origin at some time in the interval $[0, t]$ is $M_{t}^{(\varepsilon)}$-null. If $\omega$ does not hit zero, then there exists finitely many subintervals $I$ of $[0, t]$, for which there exist numbers $0<b_{I}<a_{I}$, such that $s \mapsto q(\omega(s))$ is the restriction of one of the functions $s \mapsto q\left(a_{I}-s\right), s \leq b_{I}$ or $s \mapsto q\left(a_{I}+s\right), s \geq 0$ to $I$. Because $q$ is assumed to be locally integrable on $\mathbb{R}_{++}$, each of these functions is integrable over each interval $I$, and so $q \circ \omega$ is integrable over $[0, t]$ itself.

The semigroups $e^{i\left(\tau_{k, \varepsilon} \dot{-q}\right) t}, t \geq 0, k \in \mathbb{Z}, k \neq 0$, and hence, $e^{i\left(D_{\varepsilon} \dot{-} Q_{r}(q)\right) t}, t \geq 0$, may be represented as a perturbation series expansion in terms of the semigroup given by expression (5.1).

Theorem 5.2. Let $q: \mathbb{R}_{++} \rightarrow \mathbb{R}$ be a locally square integrable function and $t>0$. Then for every $\varepsilon>0$, the function $\omega \mapsto e^{-i j_{0}^{t} q(\omega(s)) d s}$ defined for $M_{t}^{+}$-almost all $\omega \in \Omega$ is $M_{t}^{(\varepsilon)}$-integrable, and the equality

$$
e^{i\left(D_{\varepsilon} \dot{-} Q_{r}(q)\right) t}=\int_{\Omega} e^{-i \int_{0}^{t} q(\omega(s)) d s} d M_{t}^{(\varepsilon)}(\omega)
$$

is valid.

Proof. Let $q_{n}=q \chi_{\{|q| \leq n\}}$ for each $n=1,2, \ldots$. Then by dominated convergence, for each $\omega \in \Omega_{t} \backslash \Gamma_{t}$, the function $q_{n} \circ \omega$ converges to $q \circ \omega$ almost everywhere on $[0, t]$ and $\int_{0}^{t} q_{n}(\omega(s)) d s$ converges to $\int_{0}^{t} q(\omega(s)) d s$. An appeal to dominated convergence for the operator valued measure $M_{t}^{(\varepsilon)}$ proves that the operators $\int_{\Omega} e^{-i \int_{0}^{t} q_{n}(\omega(s)) d s} d M_{t}^{(\varepsilon)}(\omega), n=1,2, \ldots$ converge in the strong operator topology to the right hand side of (5.2).

Another application of dominated convergence to the expression (5.1) and the perturbation expansion of $e^{\tau(q)+U_{k, \varepsilon}}$ in terms of the bounded matrix 
multiplication operator $U_{k, \varepsilon}[\mathrm{K} 1$, Theorem IX.2.1] shows that the operators $e^{i\left(\tau_{k, \varepsilon}-q_{n}\right) t}, n=1,2, \ldots$ converge in the strong operator topology to $e^{i\left(\tau_{k, \varepsilon} \dot{-} q\right) t}$, hence the operators $e^{i\left(D_{\varepsilon}-Q_{r}\left(q_{n}\right)\right) t}, n=1,2, \ldots$ converge in the strong operator topology to $e^{i\left(D_{\varepsilon}-Q_{r}(q)\right) t}$. The equality (5.2) is true for bounded measurable functions $q$ by virtue of [J2, Lemma 6.2] and formula (3.2). Therefore, it is true for all locally square integrable functions $q: \mathbb{R}_{++} \rightarrow \mathbb{R}$.

The following result is the analogue of $[\mathrm{J} 2$, Lemma 2.5] in the present context.

Lemma 5.3. Suppose that $q: \mathbb{R}_{++} \rightarrow \mathbb{R}$ is a function such that the bound (0.2) holds with $\mu<\sqrt{3} / 2$. Let $q_{n}=q \chi_{|q| \leq n}$ for each $n=1,2, \ldots$ and let $k$ be a nonzero integer.

Then for every $\lambda>0$, the function $\varepsilon \mapsto\left(\lambda-i\left(\tau_{k, \varepsilon}-q_{n}\right)\right)^{-1}, 0 \leq \varepsilon \leq 1$ is continuous on the strong operator topology of $\mathscr{L}\left(L^{2}\left(\mathbb{R}_{+} ; \mathbb{C}^{2}\right)\right)$, uniformly for all $n=1,2, \ldots$.

Furthermore, as $\varepsilon \rightarrow 0^{+}$, the operators $e^{i\left(\tau_{k, \varepsilon}-q_{n}\right) t}$ converge to $e^{i\left(\tau_{k}-q_{n}\right) t}$ in the strong operator topology of $\mathscr{L}\left(L^{2}\left(\mathbb{R}_{+} ; \mathbb{C}^{2}\right)\right)$, uniformly for all $n=1,2, \ldots$, and all numbers $t$ in compact subsets of $\mathbb{R}_{+}$. As $n \rightarrow \infty$, the operators $e^{i\left(\tau_{k, \varepsilon}-q_{n}\right) t}$ converge in the strong operator topology of $\mathscr{L}\left(L^{2}\left(\mathbb{R}_{+} ; \mathbb{C}^{2}\right)\right)$, uniformly for all $\varepsilon>0$, and all numbers $t$ in compact subsets of $\mathbb{R}_{+}$.

Proof. Let $X$ be the Banach space of all continuous functions $f$ from $[0,1]$ to $L^{2}\left(\mathbb{R}_{+} ; \mathbb{C}^{2}\right)$ with the uniform norm $\|f\|_{\infty}=\sup _{0 \leq \varepsilon \leq 1}\|f(\varepsilon)\|_{2}$. For each $t \geq 0$, the operator $S(t): X \rightarrow X$ is defined by $(S(t) f)(\varepsilon)=e^{i \tau_{k, \varepsilon}} f(\varepsilon)$ for $f \in X$ and $0 \leq \varepsilon \leq 1$. Then, as in the proof of [J2, Lemma 2.5], $S$ is a contraction $\mathrm{C}_{0}$-semigroup of operators acting on $X$. The contraction property follows from the Trotter product formula, for example, and the observation that $U_{k, \varepsilon}(r)$ is hermitian for each $r>0$.

The operator $\tau_{k, \varepsilon} \doteq q$ is equal to $\tau(q)+U_{k, \varepsilon}$, so applying dominated convergence to the expression (5.1) and the perturbation expansion of $e^{i\left(\tau(q)+U_{k, \varepsilon) t}\right.}$ in terms of the bounded matrix multiplication operator $U_{k, \varepsilon}$ (see the proof of [K, Theorem IX.2.1]), we see that the mapping $\varepsilon \rightarrow e^{i\left(\tau_{k, \varepsilon} \dot{-} q\right) t}$, $\varepsilon>0$, is continuous on the open interval $(0, \infty)$ in the strong operator topology of $\mathscr{L}\left(L^{2}\left(\mathbb{R}_{+} ; \mathbb{C}^{2}\right)\right)$, uniformly for $t$ in compact subsets of $\mathbb{R}_{++}$. That $e^{i\left(\tau_{k, \varepsilon} \dot{-} q\right) t}$, $t \geq 0$, is a contraction semigroup follows from the Trotter product formula. The question of continuity at $\varepsilon=0$ remains.

A result originating with $\mathrm{F}$. Rellich [G-R], [Ka, Theorem 5] ensures that $\tau_{k}-q$ is essentially selfadjoint on $C_{c}^{\infty}\left(\mathbb{R}_{++}, \mathbb{C}^{2}\right)$ if $\mu<\sqrt{3} / 2$. Its closure in $L^{2}\left(\mathbb{R}_{+} ; \mathbb{C}^{2}\right)$ is denoted by the same symbol. Trotter's convergence theorem [T, Theorem 5.2] shows that $\lim _{\varepsilon \rightarrow 0^{+}} e^{i\left(\tau_{k, \varepsilon}-q\right) t}=e^{i\left(\tau_{k}-q\right) t}$ in $\mathscr{L}\left(L^{2}\left(\mathbb{R}_{+} ; \mathbb{C}^{2}\right)\right)$, uniformly for $t$ in compact subsets of $\mathbb{R}_{+}$. For each $t \geq 0$, and function $q$ satisfying $(0.2)$, the operator $S_{q}(t)$ is defined for each $f \in X$, by 


$$
\left(S_{q}(t) f\right)(\varepsilon)=e^{i\left(\tau_{k, \varepsilon}-q\right) t} f(\varepsilon), \quad \text { for all } 0 \leq \varepsilon \leq 1 .
$$

Then $S_{q}$ is a contraction $\mathrm{C}_{0}$-semigroup acting on $X$. The contraction property is immediate from the earlier observation that $e^{i\left(\tau_{k, \varepsilon}-q\right) t}, t \geq 0$, is a contraction semigroup for each $\varepsilon>0$. Continuity at zero is valid on the dense subspace $C([0,1]) \otimes L^{2}\left(\mathbb{R}_{+} ; \mathbb{C}^{2}\right)$ of $X$; the uniform boundedness of $S_{q}$ ensures that continuity at zero is valid on all of $X$.

Now $C_{c}^{\infty}\left(\mathbb{R}_{++} ; \mathbb{C}^{2}\right)$ is a core for $\tau_{k}-q$, hence, $C([0,1]) \otimes C_{c}^{\infty}\left(\mathbb{R}_{++} ; \mathbb{C}^{2}\right)$ is a core for the infinitesimal generator $\tau_{k, \cdot}-q$ of $S_{q}$, because for any Banach space $B$, the linear space $C([0,1]) \otimes B$ is dense in the Banach space $C([0,1], B)$ equipped with the uniform norm. Another appeal to Trotter's convergence result [T, Theorem 5.2] implies that as $n \rightarrow \infty, S_{q_{n}}(t) \rightarrow S_{q}(t)$ in $\mathscr{L}(X)$, uniformly for $t$ in compact subsets of $\mathbb{R}_{+}$.

Let $\lambda>0$. For each $h \in L^{2}\left(\mathbb{R}_{+} ; \mathbb{C}^{2}\right)$ and $n=1,2, \ldots$, the function

$$
\int_{0}^{\infty} e^{-\lambda t} S_{q_{n}}(t)(\mathbb{1} \otimes h) d t
$$

is just the continuous mapping $\varepsilon \mapsto\left(\lambda-i\left(\tau_{k, \varepsilon}-q_{n}\right)\right)^{-1} h$, where the function identically equal to one on $[0,1]$ has been denoted by 1 . As $n \rightarrow \infty$, these functions converge uniformly on $[0,1]$ to the function $\varepsilon \mapsto\left(\lambda-i\left(\tau_{k, \varepsilon}-q\right)\right)^{-1} h$, proving the first conclusion. The second follows from the existence of the limit $\lim _{n \rightarrow \infty} S_{q_{n}}(t)(\mathbb{1} \otimes h)=S_{q}(t)(\mathbb{1} \otimes h)$ in $X$.

In the following theorem, we finally prove the validity of the Feynman representation $(0.1)$.

Theorem 5.4. Suppose that $q: \mathbb{R}_{++} \rightarrow \mathbb{R}$ is a function such that the bound (0.2) holds with $\mu<\sqrt{3} / 2$. Let $t>0$. Then the function $\omega \mapsto e^{-i j_{0}^{t} q(\omega(s)) d s}$ defined for $M_{t}^{+}$-almost all $\omega \in \Omega$ is $M_{t}^{+}-M_{t}$-integrable.

The operator $D-Q_{r}(q)$ is essentially selfadjoint on $C_{c}^{\infty}\left(\mathbb{R}^{3} \backslash\{0\} ; \mathbb{C}^{4}\right)$. Let $H$ denote the closure of $-D+Q_{r}(q)$ in $L^{2}\left(\mathbb{R}^{3} ; \mathbb{C}^{4}\right)$. Then the equality

$$
e^{-i H t}=\int_{\Omega} e^{-i \int_{0}^{t} q(\omega(s)) d s} d M_{t}(\omega)
$$

is valid.

Proof. Let $q_{k}=q \chi_{|q| \leq k}, k=1,2, \ldots$ By [J2, Lemma 6.2], for each $k=$ $1,2, \ldots$, the function $\omega \mapsto e^{-i j_{0}^{t} q_{k}(\omega(s)) d s}$ is $M_{t}^{+}-M_{t}$-integrable and

$$
e^{i\left(D-Q_{r}\left(q_{k}\right)\right) t}=\int_{\Omega} e^{-i \int_{0}^{t} q_{k}(\omega(s)) d s} d M_{t}(\omega) .
$$

We shall show that $e^{-i \int_{0}^{t} q_{k^{\circ} X_{s} d s}}$ converges to $e^{-i \int_{0}^{t} q^{\circ} X_{s} d s}$ in $L^{1}\left(M_{t}^{+}, M_{t}\right)$, as $k \rightarrow \infty$. 
By Theorem 5.2, $e^{-i \int_{0}^{t} q^{\circ} X_{s} d s}$ is $M_{t}^{+}$-integrable, and dominated convergence ensures that the function $e^{-i \int_{0}^{t} q_{k^{\circ}} X_{s} d s}$ converges to $e^{-i \int_{0}^{t} q^{\circ} X_{s} d s}$ in $L^{1}\left(M_{t}^{+}\right)$, as $k \rightarrow \infty$. According to [J2, Proposition 5.7], it remains to prove that for each $E \in \mathscr{S}_{t}$, the operators

$$
\int_{E} e^{-i \int_{0}^{t} q_{k^{\circ}} X_{s} d s} d M_{t}^{(\varepsilon)}, k=1,2, \ldots
$$

converge in the weak operator topology, uniformly for $\varepsilon>0$.

For each elementary event $E$ of the form (1.1), the operators (5.5) may be represented in the form (1.2), with $S$ equal to the semigroup $e^{-i\left(D_{\varepsilon}-Q_{r}\left(q_{k}\right)\right)}$. and with $Q$ equal to the spectral measure $Q_{r}$. To see this write

$$
\begin{aligned}
& \exp \left[-i \int_{0}^{t} q_{k} \circ X_{s} d s\right]= \exp \left[-i \int_{t_{n}}^{t} q_{k} \circ X_{s} d s\right] \cdots \exp \left[-i \int_{0}^{t_{1}} q_{k} \circ X_{s} d s\right] \\
&=\exp \left[-i \int_{0}^{t-t_{n}} q_{k} \circ X_{t_{n}+s} d s\right] \cdots \\
& \exp \left[-i \int_{0}^{t_{1}} q_{k} \circ X_{s} d s\right] .
\end{aligned}
$$

Recall that $\mathscr{S}_{t}$ is the algebra generated by all elementary events before time s. Suppose that $f_{j+1}$ is an $\mathscr{S}_{t_{j+1}-t_{j}}$-simple function, $j=0, \ldots, n$, with $t_{0}=0$ and $t_{n+1}=t$. For each $s \geq 0$, let $\theta_{s}: \Omega \rightarrow \Omega$ be the shift map defined by $\theta_{s}(\omega)(r)=\omega(r+s)$, for all $r \geq 0$ and $\omega \in \Omega$. The function $f_{n+1} \circ \theta_{t_{n}} \cdots f_{2} \circ \theta_{t_{1}} f_{1}$ is $\mathscr{I}_{t}$-simple and because $M_{s}^{(\varepsilon)}, s \geq 0$, are measures associated with an $\left(S_{D_{\varepsilon}}, Q_{r}\right)$-process, we have

$$
\begin{aligned}
& \int_{E} f_{n+1} \circ \theta_{t_{n}} \cdots f_{2} \circ \theta_{t_{1}} f_{1} d M_{t}^{(\varepsilon)}= \\
& \quad M_{t-t_{n}}^{(\varepsilon)}\left(f_{n+1}\right) Q_{r}\left(B_{n}\right) M_{t_{n}-t_{n-1}}^{(\varepsilon)}\left(f_{n}\right) \cdots Q_{r}\left(B_{1}\right) M_{t_{1}}^{(\varepsilon)}\left(f_{1}\right) .
\end{aligned}
$$

Dominated convergence ensures that the equality extends to the case in which the function $f_{j+1}$ is a bounded and $\mathscr{R}_{t_{j+1}-t_{j}}$-measurable function for each $j=0, \ldots, n$. In particular, if we choose $f_{j+1}=\exp \left[-i \int_{0}^{t_{j+1}-t_{j}} q_{k} \circ X_{s} d s\right]$, $j=0, \ldots, n$, then by Theorem 5.2, the operator (5.5) becomes

$$
e^{-i\left(D_{\varepsilon}-Q_{r}\left(q_{k}\right)\right)\left(t-t_{n}\right)} Q_{r}\left(B_{n}\right) e^{-i\left(D_{\varepsilon}-Q_{r}\left(q_{k}\right)\right)\left(t_{n}-t_{n-1}\right)} \cdots Q_{r}\left(B_{1}\right) e^{-i\left(D_{c}-Q_{r}\left(q_{k}\right)\right) t_{1}},
$$

as was to be established

An appeal to Lemma 5.3 and the Banach-Steinhaus theorem shows that the convergence of the operators (5.5) is actually in the strong operator topology, uniformly for $\varepsilon>0$. Here we need to take the direct sum of operators unitarily equivalent to the operators treated in Lemma 5.3. Hence, $e^{-i \int_{0}^{t} q^{\circ} X_{s} d s}$ is $M_{t}^{+}-M_{t}$-integrable, and the function $e^{-i \int_{0}^{t} q_{k^{\circ}} X_{s} d s}$ converges to 
$e^{-i \int_{0}^{t} q^{\circ} X_{s} d s}$ in $L^{1}\left(M_{t}^{+}, M_{t}\right)$, as $n \rightarrow \infty$

As in the proof of Lemma $5.3, D-Q_{r}(q)$ is essentially selfadjoint on $C_{c}^{\infty}\left(\mathbb{R}^{3} \backslash\{0\} ; \mathbb{C}^{4}\right)$ and the left hand side of (5.4) converges in the strong operator topology to $e^{-i H t}$ as $n \rightarrow \infty$. By [J2, Proposition 5.7], or more simply, from the definition of convergence in the space $L^{1}\left(M_{t}^{+}, M_{t}\right)$, the right hand side of (5.4) converges in the strong operator topology to $\int_{\Omega} e^{-i \int_{0}^{t} q(\omega(s)) d s} d M_{t}(\omega)$ as $n \rightarrow \infty$, thereby proving the equality (5.3).

Corollary 5.5. The selfadjoint operator $H$ above is the limit in the sense of strong resolvent convergence of $D_{\varepsilon} \dot{-} Q_{r}(q)$ as $\varepsilon \rightarrow 0^{+}$.

Proof. We proved in Lemma 5.3 that for each nonzero integer $k$, the operator $\tau_{k, \varepsilon} \dot{-} q$ converges to $\tau_{k} \dot{-} q$ in the sense of strong resolvent convergence. The result follows after taking the direct sum of operators unitarily equivalent to these.

For the case $\sqrt{3} / 2 \leq \mu<1$ in the bound (0.2), we have the following result. Essential to the proof is a convergence result of $R$. Wüst [Wü] and T. Kato [K2, Theorem II] for the Dirac operator.

Theorem 5.6. Suppose that $q: \mathbb{R}_{++} \rightarrow \mathbb{R}$ is a function such that the bound (0.2) holds with $\mu<1$, and suppose that $q^{(1)} \geq 0$ near the origin. Let $t>0$. Then the function $\omega \mapsto e^{-i \kappa \mathrm{f}_{0}^{t} q(\omega(s)) d s}$ defined for $M_{t}^{+}$-almost all $\omega \in \Omega$ is $M_{t}^{+}-M_{t}$-integrable for all $\kappa \in \mathbb{C}$ such that $\mathfrak{I}(\kappa)<0$. There exists $\varepsilon>0$ such that the map

$$
\kappa \mapsto \int_{\Omega} e^{-i \kappa \int_{0}^{t} q(\omega(s)) d s} d M_{t}(\omega), \quad \kappa \in \mathbb{C}, \mathfrak{I}(\kappa)<0,|\kappa| \leq 1+\varepsilon,
$$

is the restriction of a function continuous on the set $\{\kappa \in \mathbb{C}: \Im(\kappa) \leq 0,|\kappa| \leq 1+\varepsilon\}$, for the strong operator topology of $\mathscr{L}\left(L^{2}\left(\mathbb{R}^{3} ; \mathbb{C}^{4}\right)\right)$.

Moreover, there exists a selfadjoint operator $H$ such that the equality

$$
e^{-i H t}=\lim _{\substack{\Im(\kappa)<0, \kappa \rightarrow 1}} \int_{\Omega} e^{-i \kappa \int_{0}^{t} q(\omega(s)) d s} d M_{t}(\omega)
$$

is valid. The selfadjoint operator $H$ is characterised by the equality

$$
e^{-i H t}=\lim _{n \rightarrow \infty} e^{i\left(D-Q_{r}\left(q_{n}\right)\right) t},
$$

for all $t \in \mathbb{R}$, where $q_{n}=q \chi_{|q| \leq n}$ for each $n=1,2, \ldots$.

If $q^{(1)} \leq 0$ near the origin, then the analogous statement is valid for the region $\mathfrak{I}(\kappa) \geq 0$.

Proof. With the notation of the proof of Lemma 5.3, for each $n=1,2, \ldots$, 
the infinitesimal generator of $S_{\kappa q_{n}}$ is a $i$ nded perturbation of the generator of $S$, so there exists $\lambda_{0}>0$ such that for all $\lambda>\lambda_{0}$, the map $\kappa \mapsto R_{\kappa q_{n}}(\lambda)$ is the restriction to the real axis of a function analytic in a neighbourhood of zero in $\mathbb{C}$.

Now suppose that there exists $r_{0}>0$ such that $q^{(1)}(r) \geq 0$ for all $0<r<r_{0}$. Then for $r \geq r_{0}$, we have $\left|q^{(1)}(r)\right| \leq 1 / r_{0}$. It follows from the Trotter-Kato product formula that, for $a=1 / r_{0}+\left\|q^{(2)}\right\|_{\infty}$, the bound

$$
\left\|e^{i\left(\tau_{k, \varepsilon}-\kappa q_{n}\right) t}\right\|_{\mathscr{L}\left(L^{2}\left(\mathbb{R}_{+} ; \mathbb{C}^{2}\right)\right)} \leq e^{|\Im(\kappa)| a t},
$$

holds for all $\kappa \in \mathbb{C}$ with $\mathfrak{I}(\kappa) \leq 0$, all $\varepsilon>0$, all $t \geq 0$ and all $n=1,2, \ldots$. Clearly, the bound $\left\|S_{\kappa q_{n}}(t)\right\|_{\mathscr{L}(X)} \leq e^{|\mathfrak{I}(\kappa)| a t}$ also holds for $\mathfrak{I}(\kappa) \leq 0, n=1,2, \ldots$ and $t \geq 0$. Let $\lambda_{0}>0$. Then for all $\lambda \geq \lambda_{0}$ and $n=1,2, \ldots$, the resolvent operator

$$
R_{\kappa q_{n}}(\lambda)=\int_{0}^{\infty} e^{-\lambda t} S_{\kappa q_{n}}(t) d t
$$

of $S_{\kappa q_{n}}$ has the property that $\kappa \mapsto R_{\kappa q_{n}}(\lambda)$ is analytic in the strip $\Im(\kappa)<0$, $|\mathfrak{I}(\kappa)|<\lambda / a$ and continuous for $\mathfrak{I}(\kappa) \leq 0,|\mathfrak{I}(\kappa)|<\lambda / a$. Moreover, the bound

$$
\left\|R_{\kappa q_{n}}(\lambda)\right\|_{\mathscr{L}(X)} \leq \frac{1}{\lambda-|\mathfrak{I}(\kappa)| a}
$$

obtains for all $\mathfrak{I}(k) \leq 0,|\mathfrak{I}(\kappa)|<\lambda / a$ and $n=1,2, \ldots$.

According to [K2, Theorem I (iii)], in the case that $\mathfrak{I}(\kappa) \leq 0$ and $|\kappa|<$ $1 / 2$, the subspace $C_{c}^{\infty}\left(\mathbb{R}_{++} ; \mathbb{C}^{2}\right)$ is a core for $\tau_{k}-\kappa q$, the operator $i\left(\tau_{k}-\kappa q\right)$ is the generator of a $\mathrm{C}_{0}$-semigroup and the bound (5.7) holds with $\varepsilon=0$. Actually, the proof of [K2, Theorem I] refers to the direct sum $H(\kappa)$ of operators unitarily equivalent to $\tau_{k}-\kappa q$ for every $k= \pm 1, \pm 2, \ldots$, but we can take the restriction of $H(\kappa)$ to the reducing subspaces, because we are working with the radially symmetric potential $V: x \mapsto q(|x|), x \in \mathbb{R}^{3}$.

An argument analogous to that of Theorem 5.4 for the case of real $\kappa$ with $|\kappa|<\sqrt{3} / 2$, shows that for each $\kappa \in \mathbb{C}$ with $\mathfrak{I}(\kappa) \leq 0$ and $|\kappa|<1 / 2$, the operator $S_{\kappa q_{n}}(t)$ converges as $n \rightarrow \infty$ to the operator $S_{\kappa q}(t)$ in $\mathscr{L}(X)$, uniformly for $t$ in compact subsets of $\mathbb{R}_{+}$. An appeal to Vitali's convergence theorem [H-P, Theorem 3.14.1] ensures that $S_{\kappa q_{n}}(t), n=1,2, \ldots$ converges in the strong operator topology of $\mathscr{L}(X)$, uniformly as $\kappa$ ranges over compact subsets of the region $\mathfrak{I}(\kappa)<0$. The limiting semigroup is denoted by $S_{\kappa q}$ for all complex numbers $\kappa$ satisfying $\mathfrak{I}(\kappa)<0$. As in the proof of Theorem 5.4, the function $\omega \mapsto e^{-i \kappa \int_{0}^{t} q(\omega(s)) d s}$ is $M_{t}^{+}-M_{t}$-integrable for all $\kappa \in \mathbb{C}$ such that $\mathfrak{I}(\kappa)<0$. Furthermore, 


$$
\left[S_{\kappa q}(t)(\mathbb{1} \otimes h)\right](\varepsilon)=\int_{\Omega} e^{-i \kappa f_{0}^{t} q(\omega(s)) d s} d\left(M_{t}^{(\varepsilon)} h\right)(\omega), \quad 0 \leq \varepsilon \leq 1,
$$

for all $\Im(\kappa)<0$ and $h \in L^{2}\left(\mathbb{R}_{+} ; \mathbb{C}^{2}\right)$.

By dominated convergence, for each $\lambda>a$ and $\kappa \in \mathbb{C}$ satisfying $\Im(\kappa) \leq 0$ and $|\kappa|<\min (1 / 2, \lambda / a)$, the resolvent operator $R_{\kappa q_{n}}(\lambda)$ of $S_{\kappa q_{n}}$ converges in the strong operator topology of $\mathscr{L}(X)$, as $n \rightarrow \infty$, to the resolvent $R_{\kappa q}(\lambda)=\int_{0}^{\infty} e^{-\lambda t} S_{\kappa q}(t) d t$ of $S_{\kappa q}$.

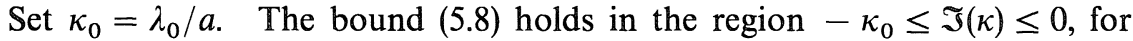
each $\lambda>\lambda_{0}$, so another appeal to Vitali's convergence theorem shows that the operator $R_{\kappa q_{n}}(\lambda), n=1,2, \ldots$ converges in $\mathscr{L}(X)$, uniformly as $\kappa$ ranges over cpmpact subsets of the open strip $\left\{\kappa \in \mathbb{C}:-\kappa_{0}<\mathfrak{I}(k)<0\right\}$.

Now suppose that $\lambda_{0}>a$ so that $\kappa_{0}=\lambda_{0} / a>1$. Then for $\lambda>\lambda_{0}, R_{\kappa q_{n}}(\lambda)$ converges in the strong operator topology to $R_{\kappa q}(\lambda)$ in the region $-\kappa_{0}<\Im(\kappa)<0$. Here, $\kappa \mapsto R_{\kappa q}(\lambda)$, defined for $-\kappa_{0}<\Im(\kappa)<0$, is the analytic continuation of $\kappa \mapsto R_{\kappa q}(\lambda)$, defined as above, for $\Im(\kappa) \leq 0$ and $|\kappa| \leq 1 / 2$. Thus, for all $\kappa \in \mathbb{C}$ such that $-\kappa_{0}<\mathfrak{I}(\kappa)<0, R_{\kappa q}(\lambda), \lambda>\lambda_{0}$ is the resolvent family of the $\mathrm{C}_{0}$-semigroup $S_{\kappa q}$. By $[\mathrm{K} 2$, Theorem $\mathbb{I}]$ and the assumption that $\mu<1$, there exists $\varepsilon>0$ such that for each $h \in L^{2}\left(\mathbb{R}_{+} ; \mathbb{C}^{2}\right)$, there exists an analytic continuation $\kappa \mapsto\left[R_{\kappa q}(\lambda)(\mathbb{1} \otimes h)\right](0)$ to all $\kappa \in \mathbb{C}$ such that $|\kappa|<1+\varepsilon$. Furthermore, by $[K 2$, Theorem II (ii)], the analytic continuation has that property that for every $h \in L^{2}\left(\mathbb{R}_{+} ; \mathbb{C}^{2}\right)$,

$$
\lim _{n \rightarrow \infty}\left[R_{\kappa q_{n}}(\lambda)(\mathbb{1} \otimes h)\right](0)=\left[R_{\kappa q}(\lambda)(\mathbb{1} \otimes h)\right](0)
$$

in $L^{2}\left(\mathbb{R}_{+} ; \mathbb{C}^{2}\right)$, and there exists a selfadjoint operator $H$, such that the operator $h \mapsto\left[R_{q}(\lambda)(\mathbb{1} \otimes h)\right](0), h \in L^{2}\left(\mathbb{R}_{+} ; \mathbb{C}^{2}\right)$ is the resolvent $(\lambda+i H)^{-1}$ of $-i H[\mathbb{K} 2$, Theorem I (iv)]. Equation (5.10) and an appeal to strong resolvent convergence [K1, Theorem IX. 2.16] shows that the operator $H$ is characterised by the stated limit.

Now for each $h \in L^{2}\left(\mathbb{R}_{+} ; \mathbb{C}^{2}\right)$, continuity ensures that

$$
\lim _{\kappa \rightarrow 1}\left[R_{\kappa q}(\lambda)(\mathbb{1} \otimes h)\right](0)=\left[R_{q}(\lambda)(\mathbb{1} \otimes h)\right](0) .
$$

By strong resolvent convergence,

$$
\lim _{\substack{\Im(\kappa)<0, \kappa \rightarrow 1}}\left[S_{\kappa q}(t)(\mathbb{1} \otimes h)\right](0)=e^{-i H t} h .
$$

Combined with formula (5.9), this establishes the equality (5.6).

Remarks. (i) In the case of the inverse square potential for the Schrödinger equation, in the region where the associated Schrödinger operator 
is not essentially selfadjoint but the Fredholm extension exists, Faris [Fa] establishes a representation similar to (5.6) with a double limit procedure.

(ii) For the Coulomb potential $q(r)=-a / r$ with $a>1$, the limit on the right hand side of (5.6) exists in the strong operator topology, but it does not define a selfadjoint operator. In the context of the Schrödinger equation, E. Nelson [N] interpreted this situation in terms of a possibility of collision with the centre of attraction. For the Dirac equation, V. Popov [P] obtains a selfadjoint extension by imposing conditions at the nuclear boundary.

Although the Feynman representation (5.3) reveals nothing about the dynamics of the Dirac particle that cannot be deduced by operator-theoretic techniques, the structure developed in the proof of the representation (5.3) may prove to be useful in situations where the quantum dynamics is not readily constructed by traditional means.

\section{References}

[A] Arai, M., On essential selfadjointness, distinguished selfadjoint extension and essential spectrum of Dirac operators with matrix valued potentials, Publ. RIMS. Kyoto Univ., 19 (1983), 33-57.

[Fa] Faris, W. G., The product formula for semigroups defined by Friedrichs extensions, Pacific. J. Math., 22 (1967), 47-70.

[G-R] Gustafson, K. E. and Rejto, P. A., Some essentially self-adjoint Dirac operators with spherically symmetric potentials, Israel J. Math., 14 (1973), 63-75.

[H-P] Hille, E. and Phillips, R. S., Functional Analysis and Semigroups, Amer. Math. Soc. Colloq. Publ. XXXI, Amer. Math. Soc., New York, 1957.

[I-T] Ichinose, T. and Tamura, H., Zitterbewegung of a Dirac particle in two-dimensional spacetime, J. Math. Phys., 29 (1988), 103-109.

[J1] Jefferies, B., Integration with respect to closable set functions, J. Funct. Anal., 66 (1986), $381-405$.

[J2] - A process associated with the radially symmetric Dirac equation, Publ. RIMS., Kyoto Univ., 30 (1994), 297-325.

[Ka] Kalf, H., Schmincke, U.-W., Walter, J. and Wüst, R., On the spectral theory of Schrödinger and Dirac operators with strongly singular potentials, Proceedings of the Symposium on Spectral Theory and Differential Equations, University of Dundee, 1974, Lect. Notes in Math. Springer-Verlag, Berlin, Heidelberg, New York, 448 (1975), $182-226$.

[K1] Kato, T., Perturbation Theory for Linear Operators, Springer-Verlag, New York, 1966.

[K2] - Holomorphic families of Dirac operators, Math. Z., 183 (1983), 399-406.

[Klu] Kluvánek, I., Operator valued measures and perturbations of semi-groups, Arch. Rat. Mech. Anal., 81 (1983), 161-180.

[K-K] Kluvánek, I. and Knowles, G., Vector Measures and Control Systems, North Holland, Amsterdam, 1976.

[N] Nelson, E., Feynman integrals and the Schrödinger equation, J. Math. Phys., 5 (1964), 332-343.

[P] Popov, V. S., "Collapse to the center" at $Z>137$ and critical nuclear charge, Soviet J. Nuclear Phys., 12 (1971), 235-243. 
[T] Trotter, H. F., Approximation of semi-groups of operators, Pacific J. Math., 8 (1958), 887-919.

[Wü] Wüst, R., Distinguished self-adjoint extensions of Dirac operators constructed by means of cut-off potentials, Math. Z., 141 (1975), 93-98. 